\title{
Induction Motor Control: Multivariable Analysis and Effective Decentralized Control of Stator Currents for High-Performance Applications
}

\author{
Luis A. Amézquita-Brooks, Member, IEEE, Jesús Licéaga-Castro, \\ Eduardo Licéaga-Castro, Member, IEEE, and Carlos E. Ugalde-Loo, Member, IEEE
}

\begin{abstract}
The adequate control of stator currents is a fundamental requirement for several high-performance induction motor (IM) control schemes. In this context, classical linear controllers remain widely employed due to their simplicity and success in industrial applications. However, the models and methods commonly used for control design lack valuable information, which is fundamental to guarantee robustness and high performance. Following this line, the design and existence of linear fixed controllers is examined using individual channel analysis and design. The studies presented here aim to establish guidelines for the design of simple (time invariant, low order, stable, minimum phase, and decentralized) yet robust and highperformance linear controllers. Such characteristics ease the implementation task and are well suited for engineering applications, making the resulting controllers a good alternative for the stator current control required for highperformance IM schemes such as field-oriented, passivitybased, and intelligent control. Illustrative examples are presented to demonstrate the analysis and controller design of an IM, with results validated in a real-time experimental platform. It is shown that it is possible to completely decouple the stator current subsystem without the use of additional decoupling elements.
\end{abstract}

Index Terms-Decentralized control, induction motors (IMs), linear feedback control systems, motor drives, realtime systems, robust multivariable control, robustness, stability.

Manuscript received August 11, 2014; revised November 21, 2014 February 15, 2015, and April 9, 2015; accepted April 12, 2015. Date of publication May 21, 2015; date of current version October 7, 2015. The work of C. E. Ugalde-Loo was supported by the Engineering and Physical Sciences Research Council, Research Councils U.K., through the Centre for Integrated Renewable Energy Generation and Supply under Grant EP/E036503/1. The data used to carry out the research reported in this article was generated as part of the Ph.D. studies by L. A. Amézquita-Brooks at the Monterrey Institute of Technology and Higher Education, Campus Estado de México, in 2010.

L. A. Amézquita-Brooks and E. Licéaga-Castro are with the Facultad de Ingeniería Mecánica y Eléctrica, Universidad Autónoma de Nuevo León, San Nicolás de los Garza 66451, Nuevo León, México (e-mail: amezquita-brooks@ieee.org; e.liceaga.c@gmail.com).

J. Licéaga-Castro is with the Universidad Autónoma Metropolitana, Azcapotzalco, Reynosa Tamaulipas, 08200, Ciudad de México, D.F., México (e-mail: ucastro21 @ hotmail.com).

C. E. Ugalde-Loo is with the Cardiff School of Engineering, Cardiff University, Cardiff, CF24 3AA, U.K. (e-mail: Ugalde-LooC@ cardiff.ac.uk).

Color versions of one or more of the figures in this paper are available online at http://ieeexplore.ieee.org.

Digital Object Identifier 10.1109/TIE.2015.2436360

\section{INTRODUCTION}

TDUCTION motors (IMs) have been historically recognized as the workhorse of the industry. Their employment as actuators provides the preferred choice for a number of industrial and research applications. Substantial innovations in power electronics and digital processing systems have enabled the use of high-performance IM control strategies. Among these, field-oriented control (FOC) has been the most popular [1]. The most successful FOC schemes aim to modify the behavior of an IM so that it resembles a dc motor, where the rotor flux and the torque are separately manipulated as they are naturally driven by different physical currents (i.e., field and armature). Since IMs do not share such a physical construction, the decoupling of the rotor flux and the torque is achieved by introducing nonlinear control elements that generate virtual flux- and torque-producing currents [1]-[3].

FOC has been traditionally implemented in two stages [1]-[3]. First, stator currents are controlled by using a voltage source inverter (VSI). The second step involves the design of a nonlinear flux-torque control law. The effectiveness of FOC is determined by the adequate decoupling, high performance, and robustness of the inner stator current control loops [4]. This is vital to reduce the burden imposed on the robustness and perturbation rejection requirements of a flux-torque controller. Other schemes not requiring the direct control of the stator currents exist (e.g., direct torque control); however, FOC schemes with a VSI actuation are the most widespread.

There are several approaches for controlling the stator current subsystem. Hysteresis controllers are easy to design and implement, but they introduce a higher level of harmonics due to changes in the switching frequency. In addition, the instantaneous error can be up to twice the hysteresis level [5], [6]. Predictive controllers have been also employed [7], but they tend to show high sensitivity to parametric variations. More importantly, a systematic procedure for the selection of weighting parameters is missing, which is counteracted by an extensive number of simulations. Other proposed methods may be too intricate for an easy industrial application. Controllers based on neural networks offer an adequate performance [8]. Nevertheless, the required computational effort represents a great disadvantage if compared with simpler alternatives.

At industrial levels, the most widely implemented schemes employ classical linear controllers due to their simplicity and 
effectiveness [9]-[11]. Through the use of controllers in a synchronous reference frame, a substantial reduction in the inherent cross-coupling effect of an IM can be achieved [12]-[14]. This is facilitated by the addition of feedforward loops, aiming to decouple the back electromotive force (EMF) voltages from the current regulation process. Moreover, by adopting a complex vector notation, the control design task may be simplified, which in turn simplifies the IM model from a multipleinput-multiple-output (MIMO) to a single-input-single-output (SISO) complex vector system [15]. This way, an analysis employing multivariable methods is avoided. Although the use of a synchronous proportional-integral (PI) controller has shown superior attributes when compared with its stationary reference frame PI counterpart, the synchronous scheme is more complex to construct [11], [14].

Regardless of the reference frame, the typical design of decentralized fixed linear controllers is often accompanied by the following:

- On-site adjustment. Controller gains are commonly adjusted by trial and error or experimental responses combined with heuristic methods [1], [9]. To address such a shortcoming, a tuning method has been suggested to achieve a response with a minimum settling time and a negligible overshoot. Such an approach sets the openloop crossover frequency to $4 \%$ of the sampling frequency [16]. In [11], a PI controller tuning procedure is proposed, where the proportional gain and integrator reset times are set to their maximum possible values. More recently, the work in [17] has proposed a MIMO rootlocus-based approach that minimizes the controller time constant and, thus, the settling time. Although the aforementioned methods may limit controller retuning, their recent publication prevents them from being widespread practices.

- Oversimplified models for control design. Although it is claimed that several simplified models capture the fundamental IM dynamics, the majority fail to include the MIMO nature of the process, the process dependence on the rotor speed, and the rotor components [10], [11].

- Decoupling networks. These aim to transform the MIMO process into SISO systems through the cancelation of the coupling [18]. Satisfactory responses have been reported using these networks and are currently considered a good high-performance option [11], [19], [20]. However, good performance has been also achieved in electric machine FOC-based applications without their use [11], [21].

The main goal of this paper is to present clear and simple control design guidelines for stationary reference frame controllers. These principles are based on a profound study of the stator current subsystem and supported by previous work. For instance, in [22] and [23], the theoretical foundation for evaluating electric machines using MIMO tools is presented, and in [24], the simplification of an IM electric subsystem was studied; in [25], the problem of torque-speed-position control is addressed. In this paper, it is shown through theoretical analysis and experiments how it is possible to design robust highperformance linear controllers as long as a few simple protocols are followed. Due to the analysis presented, a designer is not required to repeat all the theoretical assessments and may only follow the control design method. The principal characteristics of this paper can be summarized as follows:

- A full IM model including both the stator and rotor dynamics is adopted (i.e., the rotor effects are not neglected).

- The multivariable nature of the process is considered without any simplifications, direct cancelations, or information loss. Although a number of MIMO studies can be found in literature, direct cross-coupling cancelations are commonly used. The approach presented here allows evaluating and quantifying the robustness of decoupling schemes, including the resulting coupling level of the stator currents.

- The effect of the rotor angular speed is fully considered.

The omission of the rotor dynamics, the multivariable effects, and the rotor speed is common practice [10], [11]. Nonetheless, it will be shown that this is only valid if a high-bandwidth controller is used. In particular, when designing controllers that operate under a limited bandwidth, the combined effect of these factors is only negligible if proper care is exercised during the control design task. In this line, the problem is analyzed here using individual channel analysis and design (ICAD), which is a frequency-domain framework that allows the design of robust linear controllers for MIMO systems [26]. Several applications using ICAD have been reported, including satisfactory results in the analysis of the cross coupling of electrical systems [27]-[29]. In this paper, the formal analysis afforded by the ICAD framework is presented. In addition, an experimental test bench is used to demonstrate, in real time, the performance of simple controllers designed with ICAD.

The scope of this paper is limited to the analysis and control system design of stationary reference frame controllers. Through the approach followed, it is shown that a simple decentralized controller decouples the stator currents without the use of additional control structures. This simplifies the control system, which is a valuable asset in any industrial application. Moreover, it is shown that an adequately designed stationary reference frame controller is sufficient to achieve high performance, which contrasts with the responses obtained with stationary PI controllers. This is revealed through theoretical analysis and confirmed via real-time experimentation. It should be highlighted that the theoretical analysis presented here is valid for any IM regardless of its rating and parameters.

\section{Stationary Reference Frame im Model}

A classical bipolar IM model is given by [10]

$$
\begin{aligned}
\dot{\mathbf{x}} & =\mathbf{A} \mathbf{x}+\mathbf{B u} \\
\mathbf{y} & =\mathbf{C} \mathbf{x}+\mathbf{D u} \\
\tau_{E} & =\frac{3}{2}\left(\frac{P}{2}\right) \frac{L_{m}}{L_{r}}\left[\psi_{\alpha r} i_{\beta s}-\psi_{\beta r} i_{\alpha s}\right] \\
\frac{d}{d t} \omega_{r} & =\frac{P}{2 J}\left(\tau_{E}-\tau_{L}\right)
\end{aligned}
$$


with

$$
\begin{aligned}
& \mathbf{A}=\left[\begin{array}{cccc}
a_{11} & 0 & \frac{L_{m} R_{r}}{\sigma L_{s} L_{r}^{2}} & \frac{L_{m} \omega_{r}}{\sigma L_{s} L_{r}} \\
0 & a_{22} & -\frac{L_{m} \omega_{r}}{\sigma L_{s} L_{r}} & \frac{L_{m} R_{r}}{\sigma L_{s} L_{r}^{2}} \\
\frac{L_{m} R_{r}}{L_{r}} & 0 & -\frac{R_{r}}{L_{r}} & -\omega_{r} \\
0 & \frac{L_{m} R_{r}}{L_{r}} & \omega_{r} & -\frac{R_{r}}{L_{r}}
\end{array}\right] \quad \mathbf{D}=\mathbf{0}_{2 \times 2} \\
& \mathbf{B}=\left[\begin{array}{cccc}
\frac{1}{\sigma L_{s}} & 0 & 0 & 0 \\
0 & \frac{1}{\sigma L_{s}} & 0 & 0
\end{array}\right]^{T} \quad \mathbf{C}=\left[\begin{array}{llll}
1 & 0 & 0 & 0 \\
0 & 1 & 0 & 0
\end{array}\right] \\
& \mathbf{x}=\left[\begin{array}{llll}
i_{\alpha s} & i_{\beta s} & \psi_{\alpha r} & \psi_{\beta r}
\end{array}\right]^{T} \quad \mathbf{u}=\left[\begin{array}{lll}
v_{\alpha s} & v_{\beta s}
\end{array}\right]^{T} \\
& \mathbf{y}=\left[\begin{array}{ll}
i_{\alpha s} & i_{\beta s}
\end{array}\right]^{T} \\
& a_{11}=a_{22}=-\frac{L_{r}^{2} R_{s}+L_{m}^{2} R_{r}}{\sigma L_{s} L_{r}^{2}} \quad \sigma=1-\frac{L_{m}^{2}}{L_{s} L_{r}}
\end{aligned}
$$

where $i_{\alpha s}$ and $i_{\beta s}$ are the stator currents; $\psi_{\alpha r}$ and $\psi_{\beta r}$ are the rotor fluxes; $\omega_{r}$ is the rotor angular velocity; $v_{\alpha s}$ and $v_{\beta s}$ are the stator voltages; $L_{s}, L_{r}$, and $L_{m}$ are the stator, rotor, and mutual inductances, respectively; $R_{s}$ and $R_{r}$ are the stator and rotor resistances, respectively; $J$ is the rotor inertia; $\tau_{L}$ is the external load torque; $\tau_{E}$ is the generated torque; and $P$ is the number of poles. The model has been arranged so that the electrical subsystem is contained in state space realization (1). The equations in (2) represent the mechanical subsystem. The control variables of interest are $\tau_{E}, \omega_{r}$, and the rotor position $\theta_{r}$ (integral of $\omega_{r}$ ). However, a successful IM control scheme first requires the control of the stator currents by driving the stator voltages (i.e., the control inputs) using a VSI.

Although the system in (1) and (2) is nonlinear, $\omega_{r}$ varies at speeds well below the closed-loop current subsystem. This bandwidth separation allows considering (1) as linear time invariant (LTI), making it possible to design a linear controller robust to the variations of $\omega_{r}$. This is a well-known accepted property of some nonlinear systems [30]. Thus, (1) can be represented as

$$
\left[\begin{array}{l}
i_{\alpha s}(s) \\
i_{\beta s}(s)
\end{array}\right]=\left[\begin{array}{ll}
g_{11}(s) & g_{12}(s) \\
g_{21}(s) & g_{22}(s)
\end{array}\right]\left[\begin{array}{l}
v_{\alpha s}(s) \\
v_{\beta s}(s)
\end{array}\right]=\mathbf{G}(s)\left[\begin{array}{l}
v_{\alpha s}(s) \\
v_{\beta s}(s)
\end{array}\right] .
$$

$\mathbf{G}(s)=\mathbf{C}(s \mathbf{I}-\mathbf{A})^{-1} \mathbf{B}+\mathbf{D}$ is a transfer function matrix, and

$$
\mathbf{G}(s)=\frac{1}{d(s)}\left[\begin{array}{ll}
n_{11}(s) & n_{12}(s) \\
n_{21}(s) & n_{22}(s)
\end{array}\right] .
$$

The elements of (4) can be found in [23].

\section{BRIEF OVERVIEW OF ICAD}

ICAD is an analysis and control design framework used to investigate the potential and limitations for the feedback design of any MIMO LTI system. Although it is based on diagonal controllers, it can be applied to any cross-coupled system [26], [31]. ICAD is an interactive process involving the required specifications, plant characteristics, and the multivariable feedback design process. A brief overview of the ICAD setup for a
$2 \times 2$ process is presented next, although it is not limited to this case [28], [32]. Let a linear $2 \times 2$ plant be represented by

$$
\begin{aligned}
\mathbf{y}(s) & =\mathbf{G}(s) \mathbf{u}(s) \\
{\left[\begin{array}{l}
y_{1}(s) \\
y_{2}(s)
\end{array}\right] } & =\left[\begin{array}{ll}
g_{11}(s) & g_{12}(s) \\
g_{21}(s) & g_{22}(s)
\end{array}\right]\left[\begin{array}{l}
u_{1}(s) \\
u_{2}(s)
\end{array}\right]
\end{aligned}
$$

where $g_{i j}(s)$ represents scalar individual transfer functions, $y_{i}(s)$ represents the outputs, $u_{i}(s)$ represents the inputs, and $r_{i}(s)$ represents the reference signals (with $i, j=1,2$ ). Let a diagonal controller matrix be

$$
\begin{aligned}
\mathbf{u}(s) & =\mathbf{K}(s) \mathbf{e}(s) \\
{\left[\begin{array}{l}
u_{1}(s) \\
u_{2}(s)
\end{array}\right] } & =\left[\begin{array}{cc}
k_{1}(s) & 0 \\
0 & k_{2}(s)
\end{array}\right]\left[\begin{array}{l}
e_{1}(s) \\
e_{2}(s)
\end{array}\right] \\
e_{i}(s) & =r_{i}(s)-y_{i}(s) .
\end{aligned}
$$

The system in (5) and (6) can be formulated without any assumption or loss of multivariable information in terms of individual channels $c_{i}(s)$ relating references $r_{i}(s)$ with outputs $y_{i}(s)$ as

$$
c_{i}(s)=\frac{y_{i}(s)}{e_{i}(s)}=k_{i}(s) g_{i i}(s)\left(1-\gamma(s) h_{j}(s)\right)
$$

with $i \neq j ; i, j=1,2$, where

$$
\begin{aligned}
\gamma(s) & =\frac{g_{12}(s) g_{21}(s)}{g_{11}(s) g_{22}(s)} \\
h_{i}(s) & =\frac{k_{i}(s) g_{i i}(s)}{1+k_{i}(s) g_{i i}(s)} .
\end{aligned}
$$

The cross-coupling relationship is given by [26]

$$
\frac{y_{i}(s)}{r_{j}(s)}=\frac{1}{1+c_{i}(s)} \cdot \frac{g_{i j}(s)}{g_{j j}(s)} \cdot h_{j}(s)=S_{i}(s) \cdot \frac{g_{i j}(s)}{g_{j j}(s)} \cdot h_{j}(s) \text {. }
$$

Equation (10) shows that the level of cross coupling depends on the sensitivity function $S_{i}(s)$ of each channel $c_{i}(s)$.

It should be emphasized that (7) and (9) are SISO relations; hence, a classical analysis is feasible. It is known that sensitivity functions assess the capabilities of a control system to reject noise, perturbations, and parametric uncertainty. Thus, by analyzing the frequency properties of the sensitivity and complementary sensitivity functions of (7) and (9), it is possible to establish a sensitivity analysis of the overall MIMO control system. This way, the control design problem reduces to the design of a SISO controller for each channel. A block diagram of a feedback system with a diagonal control is shown in Fig. 1. The equivalent scalar channels are shown in Fig. 2.

In (8), $\gamma(s)$ is called the multivariable structure function (MSF) and is key to ICAD. It is inherent to the nature of the process and reveals important characteristics on the existence of robust controllers satisfying arbitrary specifications. The MSF has the following characteristics [31]:

- It determines the dynamical characteristics of each inputoutput configuration.

- It has an interpretation in the frequency domain. 


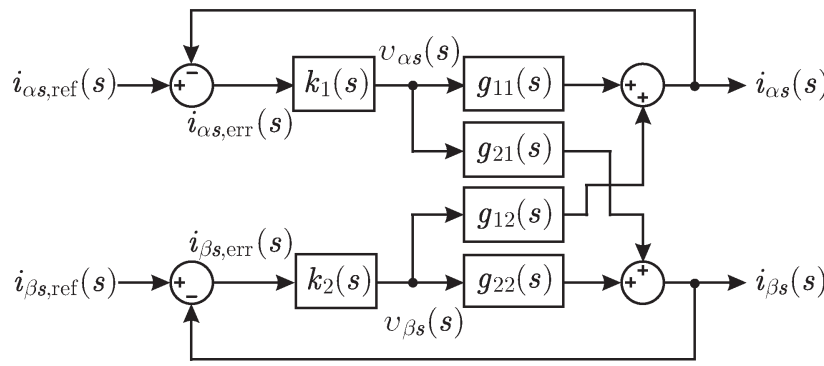

Fig. 1. MIMO $2 \times 2$ control system with a diagonal controller.

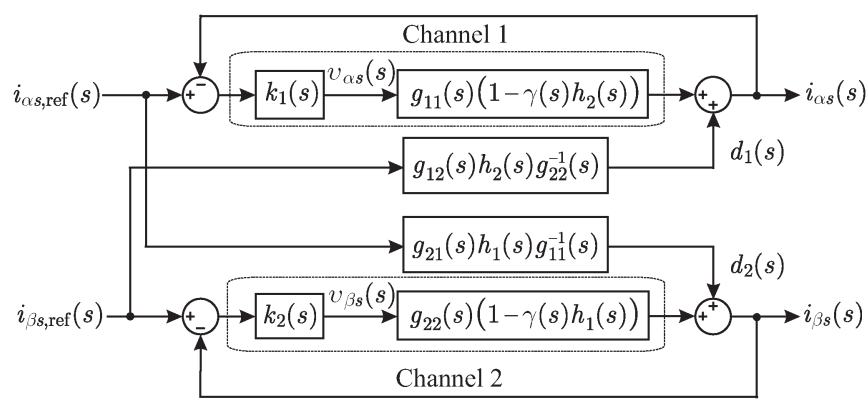

Fig. 2. Equivalent individual channel representation of a $2 \times 2$ control system.

- Its magnitude quantifies the coupling between channels.

- it is related to transmission zeros (zeros of $1-\gamma(s)=$ $\left.0=\operatorname{det}[\mathbf{G}(s)]=g_{11} g_{22}-g_{12} g_{21}\right)$. This allows testing for right-hand plane (RHP) transmission zeros using the Nyquist criterion.

- Its closeness to $(1,0)$ in the Nyquist plot indicates to what extent the plant structure (not necessarily its stability) is sensitive to uncertainty. This fact plays a key role in the design of robust controllers and allows going beyond the concept of bifurcations for nonlinear systems.

- It allows a robustness evaluation of decoupling matrices since the zeros of $(1-\gamma(s))$ are equal to the roots of $\operatorname{det}[\mathbf{G}(s)]$.

- The existence and design of stabilizing diagonal compensators can be determined from the characteristics of $\gamma(s)$. Controllers with a different structure than diagonal can be treated with slight modifications.

- It has a close relation with the relative gain array (RGA); however, the RGA is only used to define the selection of input-output pairs as a previous step to control design [33].

It is important to note that the Nyquist stability criterion and the plots of the MSF are used to evaluate the configuration robustness. Thus, the interpretation of such plots is not the same as in the classical control theory. A comprehensive treatment of this topic was presented in [26] and [31].

\section{Mimo AnAlysis: Stator CuRRENT SUBSystem}

The guidelines for the design of linear diagonal controllers for system (3) are derived in this section. The analysis, which is based on ICAD's MSF, applies to any decentralized linear control scheme. Let the individual channels be defined as

$$
\begin{aligned}
& c_{1}(s): v_{\alpha s}(s) \rightarrow i_{\alpha s}(s) \\
& c_{2}(s): v_{\beta s}(s) \rightarrow i_{\beta s}(s) .
\end{aligned}
$$

From (4) and (8), the MSF is given by

$$
\gamma(s)=\frac{n_{12}(s) n_{21}(s)}{n_{11}(s) n_{22}(s)} .
$$

The existence of stabilizing controllers for individual channels $c_{i}(s)(i=1,2)$ reduces to the existence of $k_{i}(s)$ that simultaneously stabilizes $g_{i i}(s)\left(1-\gamma(s) h_{j}(s)\right)$ and $g_{i i}(s)$ iff $\operatorname{MSF} \gamma(s)$ satisfies the following requirements [22], [26]:

(a.1) $\gamma(s)$ has no RHP poles (RHPPs)

(a.2) The Nyquist plot of $\gamma(s)$ does not encircle point $(1,0)$. (a.3) $\gamma(s)$ tends to zero as $s$ tends to infinity.

Conditions (a.1) and (a.2) ensure that $(1-\gamma(s))$ does not contain RHP zeros (RHPZs); thus, the system is minimum phase. To maintain the dynamical structure in (7), it is also required that the Nyquist plot of $\gamma(s) h_{j}(s)$ does not encircle $(1,0)$. It has been shown in [22] and [23] that all IMs defined by (1) and (2) comply with (a.1), (a.2), and (a.3) for any $\omega_{r} \in \mathbb{R}$.

For robust stability and performance, controllers $k_{i}(s)$ must comply, in addition to (a.1)-(a.3), with the following [26], [31]:

(b.1) $k_{i}(s)$ should stabilize $g_{i i}(s)\left(1-\gamma(s) h_{j}(s)\right)$ and $g_{i i}(s)$ with sufficient robustness margins.

(b.2) The Nyquist trajectories of $\gamma(s)$ and $\gamma(s) h_{j}(s)$ must not encircle nor pass near point $(1,0)$.

The stability of the control system depends on condition (b.1) only, which can be satisfied by the application of any control design technique. Condition (b.2), which is referred to as structural robustness, is unique to ICAD and is defined by the number of individual channel RHPZs and RHPPs. Recall that the transmission zeros of a $2 \times 2$ system are contained in the zeros of $(1-\gamma(s))$ (since $1-\gamma(s)=\operatorname{det}[\mathbf{G}(s)]$ ) [26], [31]. Using the Nyquist criterion for stable systems, the zeros of $(1-\gamma(s))$ are equal to the number of RHPPs of $\gamma(s)$ plus the number of encirclements to $(1,0)$ of the Nyquist plot of $\gamma(s)$. If the Nyquist plot of $\gamma(s)$ passes near $(1,0)$, then it is said that the system has low structural robustness, i.e., due to uncertainty, the number of encirclements to $(1,0)$ and, thus, the number of nonminimum-phase transmission zeros may easily change. This can be measured in terms of classical phase and gain margins.

Consider a DeLorenzo DL10115A1 three-phase squirrelcage IM. This machine has a nominal power of $300 \mathrm{~W}$, a nominal voltage of $380 \mathrm{Vrms}$, a nominal speed of $3450 \mathrm{r} / \mathrm{min}$, and 2 poles. Its state space representation (1) is given by [34]

$\mathbf{A}=\left[\begin{array}{cccc}-359.2 & 0 & 141.8 & 9.2 \omega_{r} \\ 0 & -359.2 & -9.2 \omega_{r} & 141.8 \\ 21.9 & 0 & -15.4 & -\omega_{r} \\ 0 & 21.9 & \omega_{r} & -15.4\end{array}\right] \quad \mathbf{B}=\left[\begin{array}{cc}9.7 & 0 \\ 0 & 9.7 \\ 0 & 0 \\ 0 & 0\end{array}\right]$
$\mathbf{C}=\left[\begin{array}{llll}1 & 0 & 0 & 0 \\ 0 & 1 & 0 & 0\end{array}\right] \quad \mathbf{D}=\left[\begin{array}{ll}0 & 0 \\ 0 & 0\end{array}\right]$.




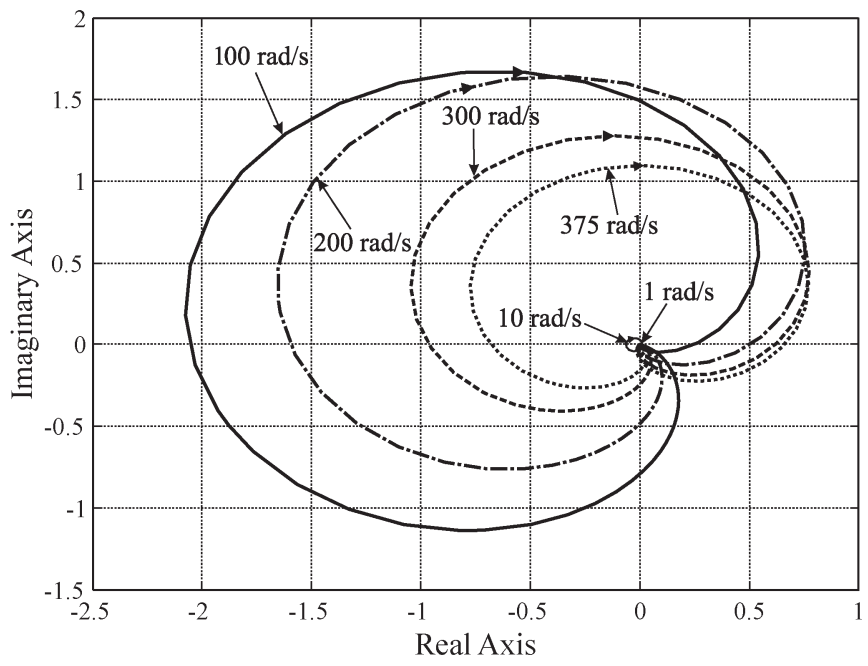

Fig. 3. Nyquist plots of $\gamma(s)$ for different values of $\omega_{r}$.

Fig. 3 shows the Nyquist plot of $\gamma(s)$ for this IM. It can be seen that the gain margin of $\gamma(s)$ decreases as rotor speed $\omega_{r}$ increases; that is, the Nyquist plot of $\gamma(s)$ passes near $(1,0)$ when $\arg [\gamma(j \omega)]=0$ for high values of $\omega_{r}$, with $\omega_{r} \in \mathbb{R}$.

It has been shown that the Nyquist plot of $\gamma(s)$ does not encircle $(1,0)$ for any IM even if it is parametrically perturbed [22], [23]. This satisfies the first part of condition (b.2). However, it is necessary to determine the proximity of $\gamma(s)$ to $(1$, $0)$ to assess the structural robustness. To this end, consider the MSF (12) evaluated at $\omega=\omega_{r}$ (i.e., $s=j \omega_{r}$ ) as follows:

$$
\gamma\left(j \omega_{r}\right)=\frac{L_{r}^{2} L_{m}^{4} \omega_{r}^{4}}{\left(\mathrm{re}_{\omega r}+j \mathrm{im}_{\omega r}\right)^{2}}
$$

where

$$
\begin{aligned}
\operatorname{re}_{\omega r} & =2 \omega_{r}^{2} \sigma L_{s} L_{r}^{2}+\omega_{r}^{2} L_{r} L_{m}^{2}-L_{r} R_{s} R_{r} \\
\operatorname{im}_{\omega r} & =-\omega_{r} \sigma L_{s} L_{r} R_{r}-2 \omega_{r} L_{r}^{2} R_{s}-\omega_{r} L_{m}^{2} R_{r} .
\end{aligned}
$$

An algebraic exercise reveals that the argument of $\gamma\left(j \omega_{r}\right)$ is

$$
\begin{array}{r}
\arg \left[\gamma\left(j \omega_{r}\right)\right]=-\tan ^{-1}\left(\frac{2 \mathrm{re}_{\omega r} \mathrm{im}_{\omega r}}{\mathrm{re}_{\omega r}^{2}-\mathrm{im}_{\omega r}^{2}}\right)-\phi \\
\phi= \begin{cases}0, & \text { for } \mathrm{re}_{\omega r}^{2}-\mathrm{im}_{\omega r}^{2} \geq 0 \\
\pi, & \text { for } \mathrm{re}_{\omega r}^{2}-\mathrm{im}_{\omega r}^{2}<0 .\end{cases}
\end{array}
$$

Additionally, we have

$$
\tan ^{-1}\left(\frac{2 \mathrm{re}_{\omega r} \mathrm{im}_{\omega r}}{\mathrm{re}_{\omega r}^{2}-\mathrm{im}_{\omega r}^{2}}\right)=\tan ^{-1}\left(\frac{-k_{1} \omega_{r}^{3}+k_{2} \omega_{r}}{k_{3} \omega_{r}^{4}-k_{4} \omega_{r}^{2}+k_{5}}\right)
$$

where $k_{1}, k_{2}, k_{3}, k_{4}$, and $k_{5}$ are real positive constants. Thus,

$$
\lim _{\omega_{r} \rightarrow \infty}\left(\frac{2 \mathrm{re}_{\omega r} \operatorname{im}_{\omega r}}{\mathrm{re}_{\omega r}^{2}-\mathrm{im}_{\omega r}^{2}}\right)=0 \Rightarrow \lim _{\omega_{r} \rightarrow \infty}\left(\arg \left[\gamma\left(j \omega_{r}\right)\right]\right)=0^{\circ} .
$$

On the other hand, the magnitude of $\gamma\left(j \omega_{r}\right)$ yields

$$
\left\|\gamma\left(j \omega_{r}\right)\right\|=\frac{L_{r}^{2} L_{m}^{4} \omega_{r}^{4}}{\sqrt{\left(\mathrm{re}_{\omega r}^{2}-\mathrm{im}_{\omega r}^{2}\right)^{2}+4 \mathrm{re}_{\omega r}^{2} \mathrm{im}_{\omega r}^{2}}} .
$$

Therefore,

$$
\begin{aligned}
\lim _{\omega_{r} \rightarrow \infty}\left\|\gamma\left(j \omega_{r}\right)\right\| & =\frac{L_{r}^{2} L_{m}^{4}}{L_{r}^{2} L_{m}^{4}+4 \sigma L_{r}^{3} L_{s} L_{m}^{2}+4 \sigma^{2} L_{r}^{4} L_{s}^{2}} \\
& =G_{M, \text { min }}<1
\end{aligned}
$$

where $G_{M \text {,min }}$ is a structural robustness measure that represents the gain margin lower bound corresponding to the highest rotor speed of any IM. By combining (17) and (19), it can be clearly noticed that the Nyquist plot of $\gamma\left(j \omega_{r}\right)$ crosses the positive part of the real axis at $\left(G_{M, \min }, 0\right)$ at a high value of $\omega_{r}$. This complies with the observation that the lowest gain margin of $\gamma(s)$ occurs at high values of $\omega_{r}$. That is, the Nyquist plot of $\gamma(s)$ is closest to point $(1,0)$ when the motor is operating at high rotor speeds (as shown in Fig. 3 ).

To comply with the second part of (b.2), the Nyquist plots of $\gamma(s) h_{j}(s)$ should be assessed along the design process of controllers $k_{i}(s)$. The following observations can help for this:

- $h_{j}(s)$ in (9) should be robust, as stated in condition (b.1).

- The bandwidth of $h_{j}(s)$ is similar to the bandwidth of the individual channels [26], [31] and should be greater than the maximum expected $\omega_{r}$; that is, the stator currents should be able to track sinusoidal signals at frequencies $\omega_{r}$.

- The gain margin lower bound $G_{M \text {, min }}$ of $\gamma(s)$ occurs at frequencies around the maximum operating $\omega_{r}$.

- The Nyquist plot of $\gamma(s) h_{j}(s)$ must not encircle $(1,0)$ for any $\omega_{r} \in \mathbb{R}$.

If these items hold, then $h_{j}\left(j \omega_{r}\right) \approx 1 \Rightarrow \gamma\left(j \omega_{r}\right) h_{j}\left(j \omega_{r}\right) \approx$ $\gamma\left(j \omega_{r}\right)$. Under a normal design scenario (if $h_{j}\left(j \omega_{r}\right) \approx 1$ and (b.1) is true), this leads to conclude, by (a.2), that $h_{j}(s)$ will not encircle $(1,0)$ since $\gamma(s)$ never does [22], [23]. This completes condition (b.2); thus, the resulting stator current control system is structurally robust. It should be emphasized that this robustness assessment is not the typical Nyquist stability criterion as, in this case, the critical point is $(1,0)$.

Let the stator current subsystem be defined by (3), controller (6), and $-\omega_{r, \max } \leq \omega_{r} \leq \omega_{r, \max }$. In agreement with the last paragraph, the following conclusions can be stated:

- The difficulty to control the system at top speed increases as $\left\|\gamma\left(j \omega_{r}\right)\right\|$ in (18) is closer to 1 for $\omega_{r}=\omega_{r, \max }$.

- $h_{j}(s)$ must have an appropriate disturbance rejection at $\omega_{r}=\omega_{r, \max }$ so that $h_{j}\left(j \omega_{r, \max }\right) \approx 1$ and that $\gamma\left(j \omega_{r, \max }\right) h_{j}\left(j \omega_{r, \max }\right) \approx \gamma\left(j \omega_{r, \max }\right)$. This is a clear requirement since the control system should track sinusoidal references at this frequency.

- If $G_{M, \min }$ in (19) approaches $(1,0)$ for high values of $\omega_{r}$, it is necessary to increase the bandwidth of $h_{j}(s)$, which is equivalent to increasing the disturbance rejection properties of $h_{j}(s)$.

- The open-loop gain requirements of controller $k_{j}(s)$ for $s=j \omega_{r}$ are determined by the closeness of $\left\|\gamma\left(j \omega_{r}\right)\right\|$ to 1 in (18).

In addition, consider a decoupling matrix $\mathbf{C}_{d}(s)$ such that $\mathbf{G}(s) \mathbf{C}_{d}(s)=\mathbf{G}_{d}(s)$, where $\mathbf{G}_{d}(s)$ is any desired decoupled 


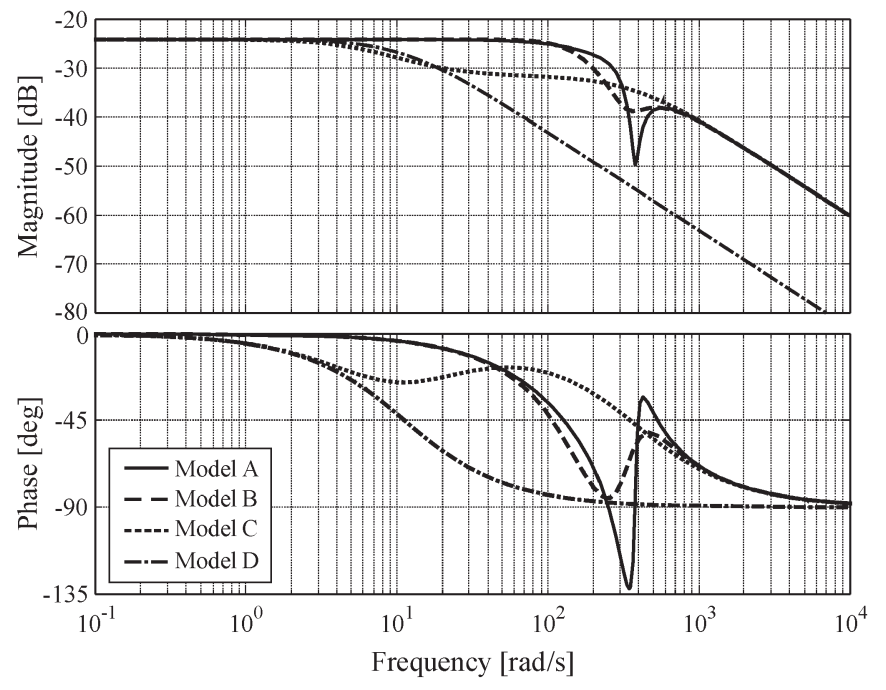

Fig. 4. Frequency response of various design models.

dynamic. Therefore, $\mathbf{C}_{d}(s)=[\mathbf{G}(s)]^{-1} \mathbf{G}_{d}(s)$. Since $[\mathbf{G}(s)]^{-1}$ exists iff $\operatorname{det}[\mathbf{G}(s)] \neq 0$ and $(1-\gamma(s))=\operatorname{det}[\mathbf{G}(s)]$, it is clear that $\gamma(s) \approx 1$ indicates a lack of robustness of a decoupling system. In this regard, (19) can be used to measure the robustness of any decoupling control system at high speeds.

The results presented in this paper demonstrate that a diagonal controller is sufficient to control the stator currents [11]. This is relevant from the viewpoint of industrial applications, where effective easy-to-implement solutions are preferred.

\section{Control Design Study Case}

The design of a stator current controller of the IM given by (13) is presented in this section as an illustrative example.

\section{A. Design Model Comparison}

When designing a controller, model oversimplification may result in a nonrobust fragile control system; thus, this should be avoided. Consider the following IM representations [24]:

- Model A: ICAD individual channel 1. This contains the multivariable nature of the system, the effect of rotor angular speed $\omega_{r}$, and all rotor components. The fullspeed range should be assessed for proper control system design.

- Model B: $n_{11}(s) / d(s)$ with an arbitrary $\omega_{r}$. The multivariable nature of the system is neglected.

- Model C: $n_{11}(s) / d(s)$ with $\omega_{r}=0 \mathrm{rad} / \mathrm{s}$. The rotor speed and multivariable nature of the system are not considered.

- Model D: $i_{\alpha s}(s) / v_{\alpha s}(s)=1 /\left(L_{s} s+R_{s}\right)$. The MIMO nature of the system, the rotor angular speed, and the rotor components are neglected. This represents the most common design model for linear stator current controllers [11].

Fig. 4 shows the frequency response of the input-output multivariable channel (Model A) compared with the responses of the alternative design models. For this exercise, $\omega_{r}=375 \mathrm{rad} / \mathrm{s}$ for Models A and B. It is evident that Model D does not provide sufficient information for designing an efficient and robust controller. Model $\mathrm{C}$ also fails in this aspect mainly because of the phase-shift error. The comparison of Models A and B demonstrates that model B lacks the important phase lag occurring at around $370 \mathrm{rad} / \mathrm{s}$. Neglecting the system phase lag has a key effect when designing robust controllers.

It is commonly considered that the effects of the rotor speed, the rotor elements, and the cross coupling are not relevant. This can be better understood by comparing Models A and D. Their main difference is the negative resonance peak coinciding with the rotor speed. Correspondingly, a phase lag effect is noted around this frequency. For both models, the phase converges to $-90^{\circ}$, whereas the gain eventually decays at $-20 \mathrm{~dB} /$ decade at higher frequencies. Thus, if a sufficiently high-gain controller is used, it could be possible to reject the effect of the negative resonance peak while maintaining stability. Under these circumstances, it seems that the additional effects in Model A are negligible. In general, it is possible to reject these effects by either increasing the overall open-loop gain or by carefully compensating for the negative resonance peak. An increase in the open-loop gain can be exercised without further plant knowledge (i.e., using Model D) but at the expense of also increasing the inverter performance requirements. Conversely, compensating for the negative resonance peak allows maintaining a lower bandwidth, but this requires the knowledge of the dynamical characteristics captured in Model A. In the following sections, a controller that exploits the additional information contained in Model A is designed.

\section{B. Control Specifications for Performance and Robustness}

A successful control strategy should be able to incorporate control specifications stated in typical electrical engineering terms. The stator current references are given by the sinusoidal signals of varying amplitudes, frequencies, and phases. In this context, the following specifications are imposed:

- Provided that the IM is under no-load conditions and that the maximum shaft speed considered is $\omega_{r, \max }=$ $375 \mathrm{rad} / \mathrm{s}$, then the closed-loop bandwidth specification is set at a frequency that is approximately a decade higher than the maximum speed. Thus, a bandwidth of at least $3300 \mathrm{rad} / \mathrm{s}$ is specified.

- Gain and phase margins over $12 \mathrm{~dB}$ and $50^{\circ}$, respectively. Such measures of robustness, which are normally used in an engineering context, can be employed for a MIMO system using ICAD.

- The cross coupling between the individual channels should be less than $-15 \mathrm{~dB}$, warranting a low coupling between the stator currents. This clear assessment of cross coupling is an easy task within ICAD and is one of its key advantages.

It is common to operate at speeds higher than that at a noload condition using field weakening techniques [10], requiring a higher bandwidth. However, in practice, the bandwidth of the stator current controller is limited by the inverter switching frequency. In this case, $5 \mathrm{kHz}$ proved to be sufficient. 


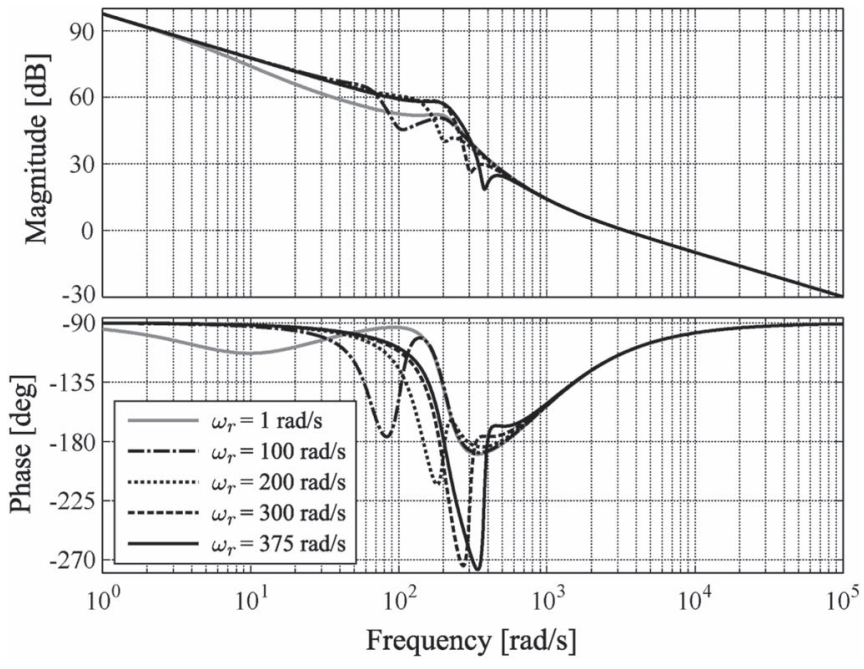

Fig. 5. Bode diagram: open-loop individual channels for different $\omega_{r}$.

\section{ICAD Controller Design}

Consider an IM (13) with individual channels defined by (11). Fig. 3 shows the Nyquist plot of $\gamma(s)$ [defined by (12)] for different $\omega_{r}$. In this figure, it is possible to confirm that the system complies with conditions (a.1)-(a.3) for $\omega_{r} \in\left[0, \omega_{r, \max }\right]$, i.e., $\gamma(s)$ has no RHPPs, the Nyquist plot of $\gamma(s)$ does not encircle $(1,0)$, and $\gamma(s) \rightarrow 0$ as $s \rightarrow \infty$. Hence, as concluded in Section IV, a stabilizing multivariable controller $k_{i}(s)$ should simultaneously stabilize $g_{i i}(s)\left(1-\gamma(s) h_{j}(s)\right)$ and $g_{i i}(s)$, with $i, j=1,2$. For robustness, the design should also comply with requirements (b.1) and (b.2).

When using ICAD, a designer must normally make the previous assessments. Nonetheless, the theoretical work in Section IV allows for the design of stator current controllers to be just centered in the stabilization of the individual channels with a bandwidth higher than the nominal rotor shaft speed. That is, controllers $k_{1}(s)=k_{2}(s)$ can be designed as a SISO controller for (7), with $h_{j}(s)=1$ and $i=1$.

A diagonal controller satisfying all the requirements is given by

$$
k_{1}(s)=k_{2}(s)=\frac{326.5(s+400)^{2}(s+1000)}{s\left(s^{2}+100 s+42500\right)}
$$

obtained using Bode shaping techniques. An integrator has been included to ensure a low steady-state error. Recall that, through (19), the gain margin lower bound for structural robustness can be assessed. This is $\approx 0.75(-2.47 \mathrm{~dB})$ for a high rotor speed. The closeness of this number to $0 \mathrm{~dB}$ indicates that $h_{i}(s)$ must feature a high disturbance rejection at $\approx 375 \mathrm{rad} / \mathrm{s}$ to ensure structural robustness. This is achieved through a high open-loop gain around this frequency. To do so, controller (20) includes a lag compensator with poles at $-50 \pm j 200$ and two zeros at -400 . To ensure stability, a sufficient phase lead is added by a zero at -1000 . It is important for the controller not to include faster dynamics than the closed-loop bandwidth as this is not suitable for a real-time implementation. The highest frequency dynamic of (20) is $1000 \mathrm{rad} / \mathrm{s}$ that is lower than the control system bandwidth, which is equal to $3350 \mathrm{rad} / \mathrm{s}$.

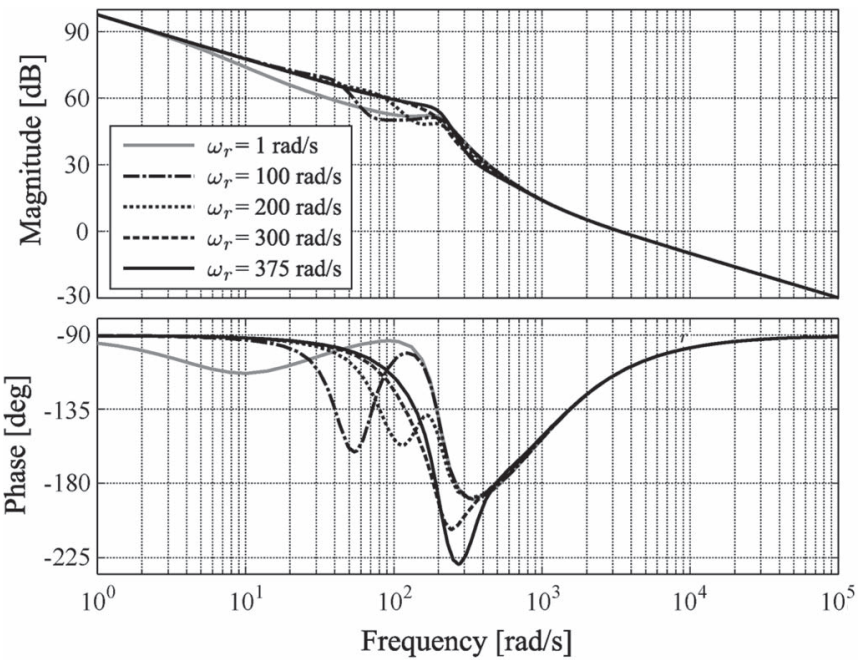

Fig. 6. Bode diagram: open-loop elements $k_{j}(s) g_{j j}(s)$ for different $\omega_{r}$.

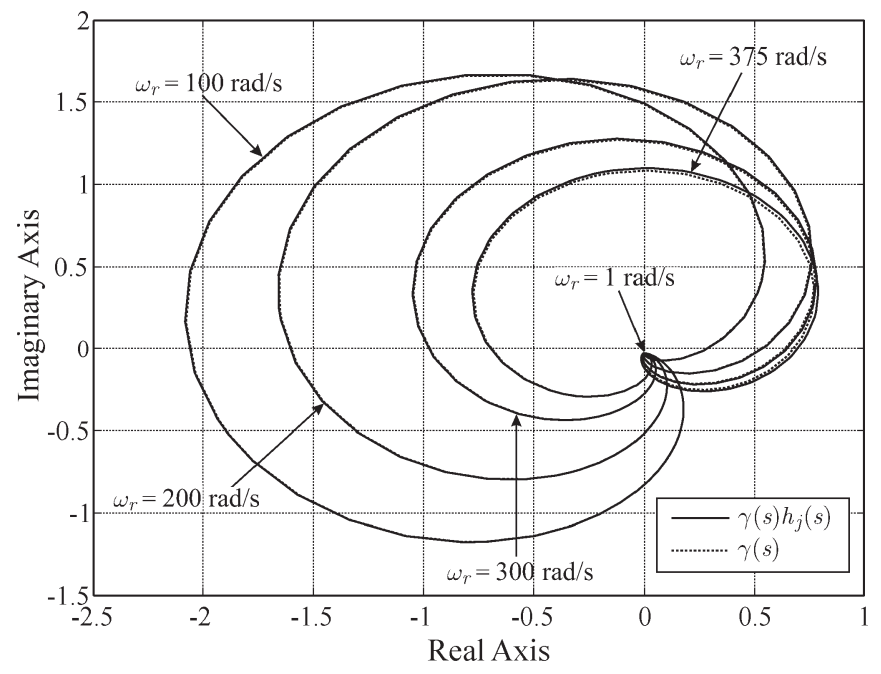

Fig. 7. Nyquist diagrams of $\gamma(s)$ and $\gamma(s) h_{j}(s)$ for different values of $\omega_{r}$.

Fig. 5 shows the robustness and performance of individual channels $c_{i}(s)$ for different values of $\omega_{r}$. Due to system symmetry, both channels are equal; the same applies to $h_{j}(s)$, $\gamma(s) h_{j}(s)$, and coupling functions (10). As it can be seen, $c_{i}(s)$ are stable and represent the overall performance of the system since the stability of the MIMO control system is determined by the stability of the individual channels [26], [31]. The robustness properties of $h_{j}(s)$ can be assessed through Fig. 6, which shows the Bode plot of $k_{i i}(s) g_{i i}(s)$ for different values of $\omega_{r}$. As it can be seen, the diagonal subsystems are robust. It can be observed that $\left|k_{i}(s) g_{i i}(s)\right|>20 \mathrm{~dB}$ around $375 \mathrm{rad} / \mathrm{s}$, ensuring a strong disturbance rejection at that frequency. The structural robustness is further assessed through the Nyquist plots of $\gamma(s) h_{j}(s)$ (see Fig. 7). Since the trajectories of $\gamma(s) h_{j}(s)$ and $\gamma(s)$ are similar and do not encircle the critical point $(1,0)$, structural robustness is ensured. The previous analysis shows that conditions (b.1) and (b.2) have been satisfied.

Table I summarizes the control system performance and robustness characteristics for an operating speed $\omega_{r}=375 \mathrm{rad} / \mathrm{s}$. The control system ensures high gain and phase margins for 
TABLE I

CONTROL SYSTEM ROBUSTNESS AND PERFormance Characteristics

\begin{tabular}{ccccccc}
\hline Measure & $c_{1}$ & $k_{1} g_{11}$ & $\gamma h_{2}$ & $c_{2}$ & $k_{2} g_{22}$ & $\gamma h_{1}$ \\
\hline Bandwidth [rad/s] & 3350 & 3350 & - & 3350 & 3350 & - \\
Gain Margin [dB] & 21 & 25.2 & 3.4 & 21 & 25.2 & 3.4 \\
Phase Margin [deg] & 67.7 & 67.7 & 46 & 67.7 & 67.7 & 46 \\
\hline
\end{tabular}

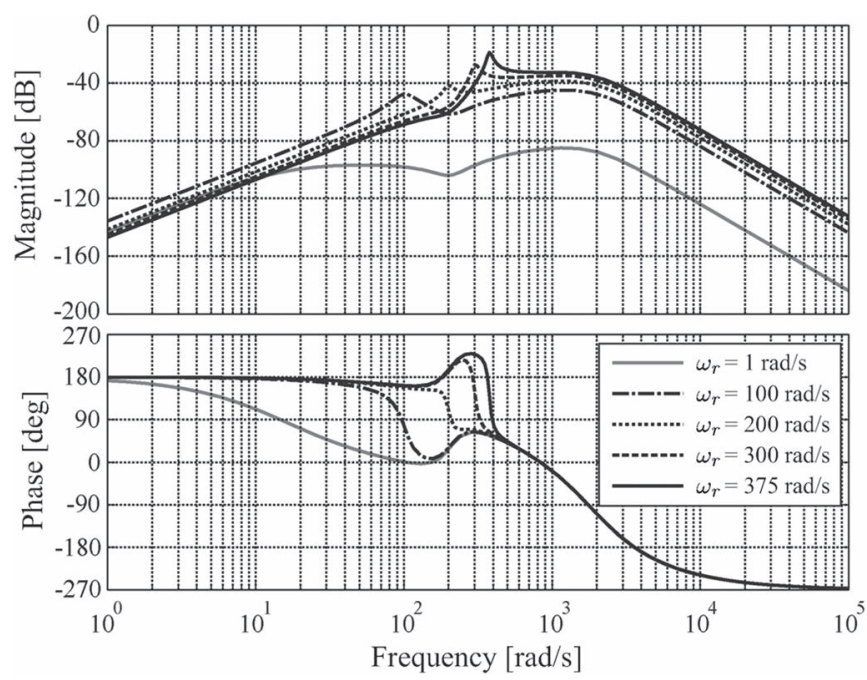

Fig. 8. Bode diagram of the coupling between the individual channels.

$c_{i}(s)$ and $k_{i i}(s) g_{i i}(s)(i=1,2)$. Although the gain margins for $\gamma(s) h_{j}(s)$ may seem low $(3.4 \mathrm{~dB})$, these have been provided for the worst case scenario (recall from (19) that the gain margin lower bound occurs at high rotor speeds).

It should be emphasized that controller (20) has stable minimum-phase low-order elements and does not contain highfrequency modes. These attributes make the designed controller well suited for engineering applications. Otherwise, the sampling frequency would be determined by the controller's highspeed modes rather than the characteristics of the process.

\section{Coupling Analysis}

The cross coupling between individual channels (see Fig. 2) can be fully assessed by (10). Fig. 8 shows the Bode plot of the coupling from input 2 to output 1 (equivalent from input 1 to output 2 due to system symmetry). In the worst case, the coupling magnitude is limited to a maximum of $-20 \mathrm{~dB}$, which complies with the specifications. Thus, the control system effectively decouples the stator currents without the use of additional decoupling elements.

\section{E. Structural Robustness to Parametric Perturbations}

A control system should be robust to parametric perturbations. Fig. 9 shows the Nyquist plot of $\gamma(s) h_{i}(s)$ under a number of critical parametric variations (at $\omega_{r}=375 \mathrm{rad} / \mathrm{s}$ ). Nominal system parameters are provided in the Appendix. It can be seen that the Nyquist plot of $\gamma(s)$ does not encircle the critical point $(1,0)$ under parametric variations. This numerically confirms that condition (a.2) has been satisfied.

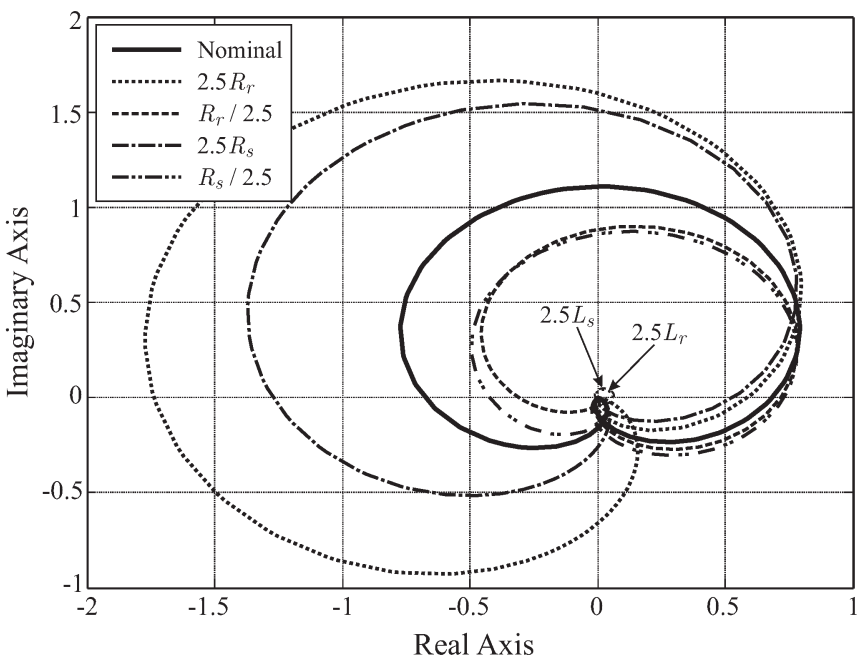

Fig. 9. Structural robustness assessment: $\gamma(s) h_{i}(s)$ under parametric perturbations $\left(\omega_{r}=375 \mathrm{rad} / \mathrm{s}\right)$.

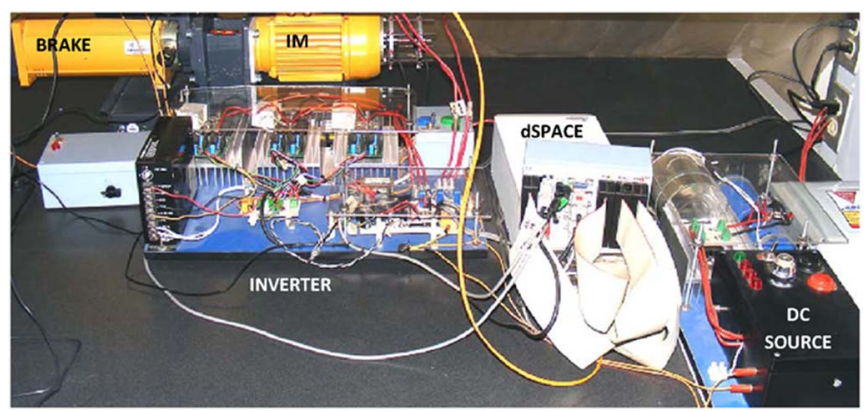

Fig. 10. Real-time experimental platform.

\section{EXPERIMENTAL RESULTS}

Real-time experiments have been performed to prove the validity of the control design in Section V. The experimental platform (see Fig. 10) consists of a DeLorenzo DL10115A1 three-phase squirrel cage IM (as in Section IV), an unregulated $310-\mathrm{V}$ dc source, a 3-phase 2-level insulated-gate-bipolartransistor-based VSI with a $5-\mathrm{kHz}$ space vector modulation, an eddy-current-based brake, and a load cell for torque measurement. It is limited to passive torque loads in speed tests (as in a brake) and to active loads in position tests. A dSPACE DS1103 digital processing system with a 50-kHz base sampling frequency was employed.

It should be noted that the proposed ICAD controller has been implemented without back-EMF compensation. Similarly, the stationary frame PI design in Section VI-D does not feature decoupling elements. The motivation behind this approach was to explore and extend the limits of pure stationary frame control, which is independent of any slip-angle estimation.

\section{A. Locked-Rotor Test}

This was performed to show the tracking capabilities of the stator current control system. Controller (20) was employed, with results shown in Fig. 11. The references for the closed-loop system $\left(i_{\alpha s}\right.$ and $\left.i_{\beta s}\right)$ consisted in sinusoids with a frequency of $300 \mathrm{rad} / \mathrm{s}$. The performance assessment is the 


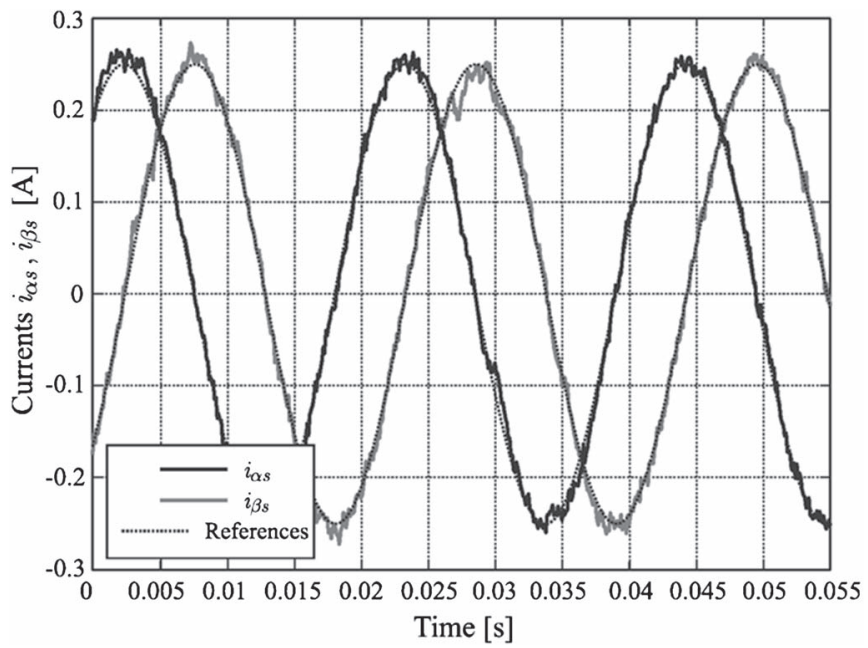

Fig. 11. Stator currents $i_{\alpha s}$ and $i_{\beta s}:$ a $300-\mathrm{rad} / \mathrm{s}$ reference with $\omega_{r}=0 \mathrm{rad} / \mathrm{s}$.

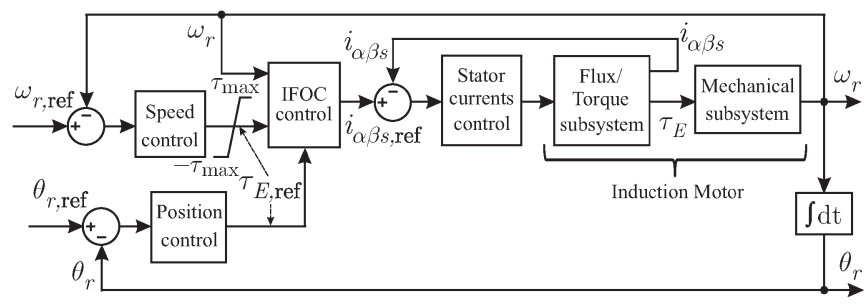

Fig. 12. Block diagram of typical FOC speed and position controllers.

successful tracking of the sinusoidal references with a low degree of error.

\section{B. Stator Current Performance: FOC Speed Controller}

An indirect FOC (IFOC) speed controller was implemented, as in [25], to evaluate the performance of the stator current subsystem under realistic conditions. The IFOC controller generates the references for the stator current controller, as shown in Fig. 12. The design and analysis of the outer speed control loop are out of the scope of this paper. For a detailed study of this component, see [25].

Fig. 13(a) shows the experimental response of the rotor speed for a step reference of $150 \mathrm{rad} / \mathrm{s}$ considering controller (20) and the FOC speed controller. An external torque load was applied along the experiment [see Fig. 13(b)]. These plots show that the FOC scheme is able to reject the torque load and maintain a good transient response. The tracking performance of the inner stator current control system can be assessed in Fig. 13(c), where both stator currents $i_{\alpha s}$ and $i_{\beta s}$ are shown together with their references. A close-up capturing the load torque change is shown, which reflects as an amplitude change in the references. The corresponding stator voltage modulation signal for phase $a$ is presented in Fig. 13(d). The signal clearly lies within the inverter modulation limits.

Once the speed reference of $150 \mathrm{rad} / \mathrm{s}$ is reached, the IM will operate under constant speed control while applying an external torque load. In general, the torque acting over the shaft is given
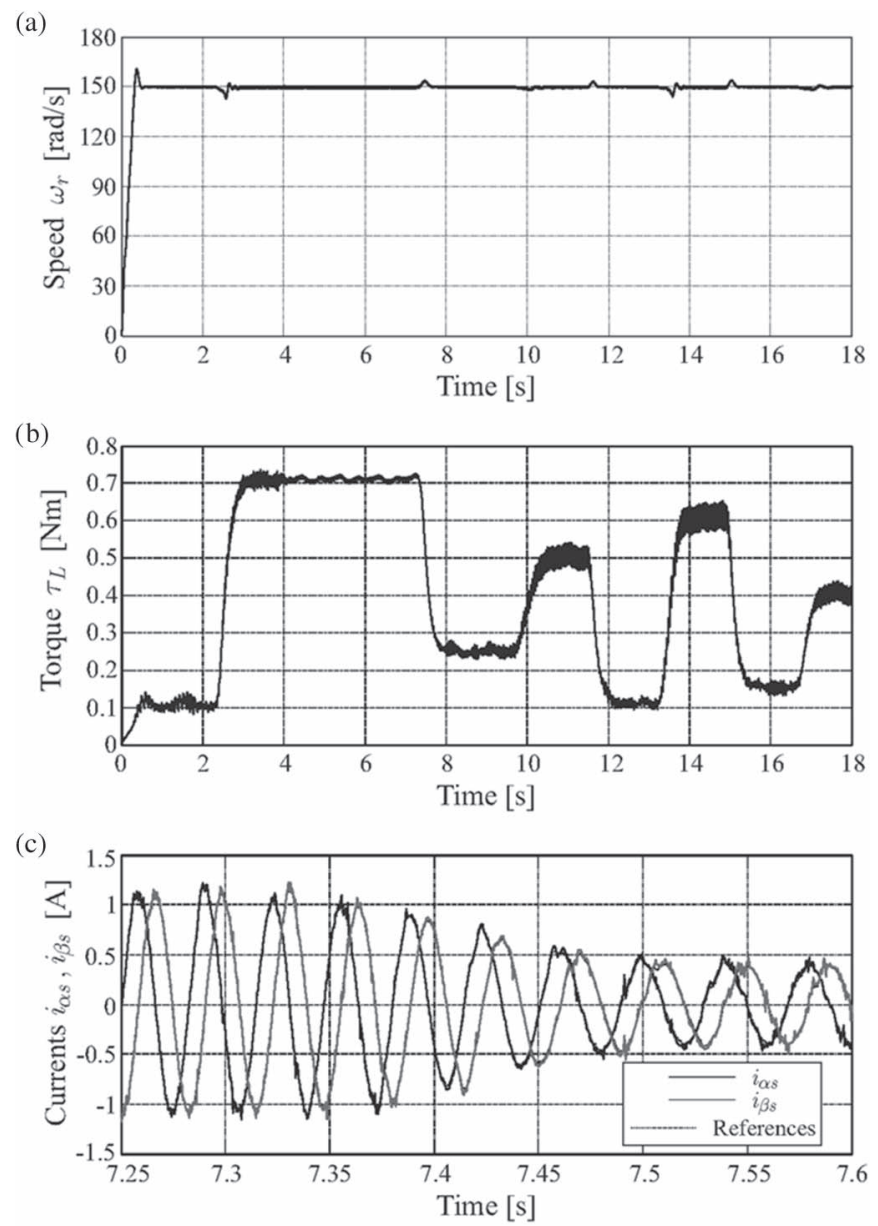

(d)

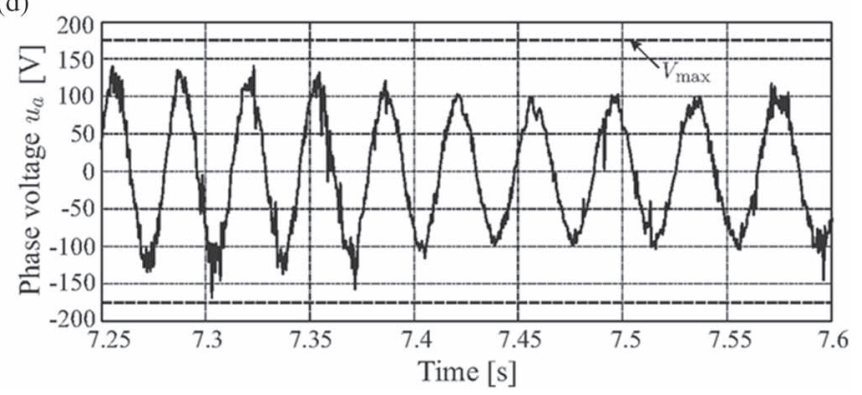

Fig. 13. Experimental responses with an IFOC speed controller and stator current controller (20) following a rotor speed reference step change. (a) Rotor angular speed $\omega_{r}$. (b) Measured load torque $\tau_{L}$. (c) Currents $i_{\alpha s} / i_{\beta s}$ and their references. (d) Phase $a$ of the stator voltage.

by $\tau_{S}=\tau_{E}-\tau_{L}-\tau_{F}$, where $\tau_{L}$ is the external torque load, $\tau_{F}$ is the shaft friction, and $\tau_{E}$ is the generated torque. Since the speed is maintained constant, $\tau_{S}=0$, and $\tau_{E}=\tau_{L}+\tau_{F}$. Thus, a change in the torque load is very similar to changing the generated torque reference in a closed loop (minus the residual friction) when operating a constant-speed closed loop.

\section{Stator Current Performance: FOC Position Controller}

Although the most common application for IMs is speed control, their use for precision servocontrol is attractive. Fig. 12 shows an IFOC position controller. This was designed, as in [25], to assess the proposed stator current controller. 
(a)

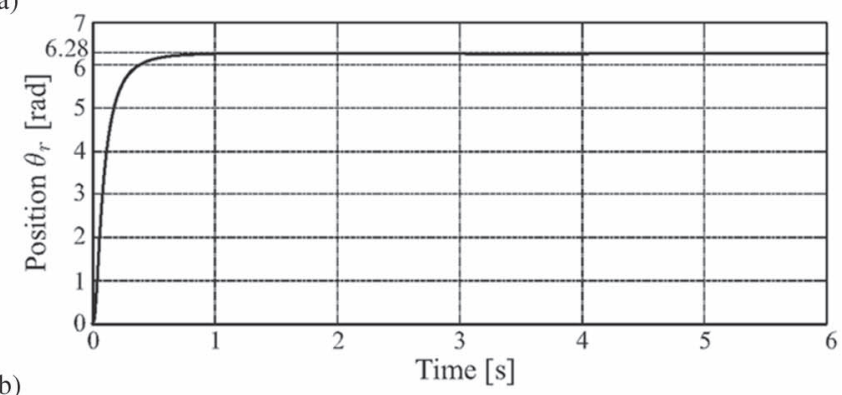

(b)

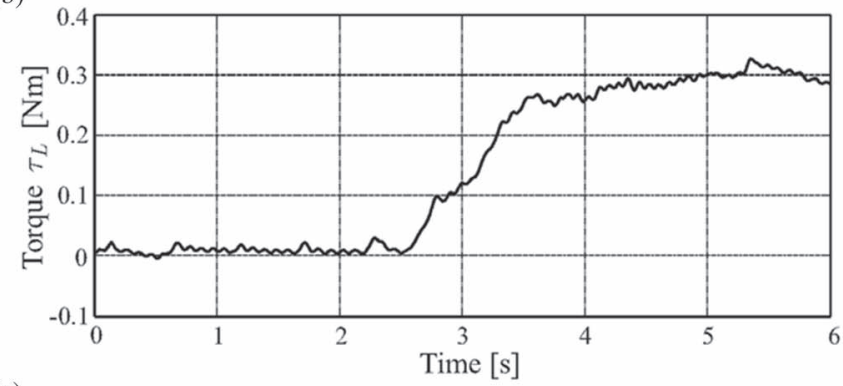

(c)

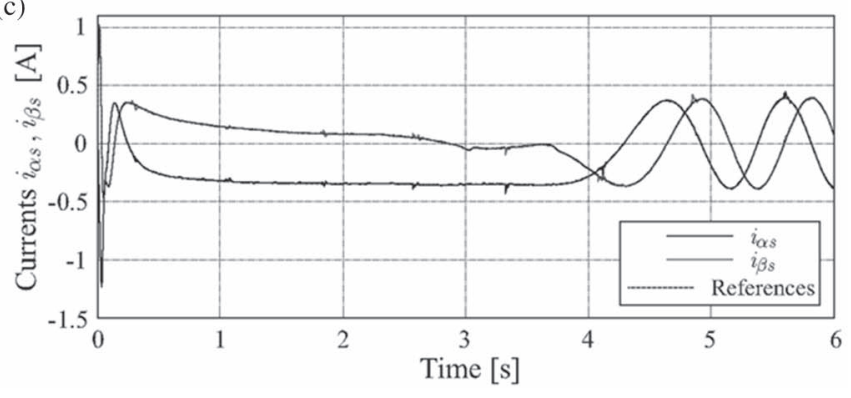

Fig. 14. Experimental responses with an IFOC position controller and stator current controller (20) following a rotor position reference step change. (a) Position $\theta_{r}$. (b) Measured load torque $\tau_{L}$. (c) Currents $i_{\alpha s} / i_{\beta s}$ and their references.

The experimental response to a step reference $(6.28 \mathrm{rad})$ is shown in Fig. 14(a). A torque load was introduced at $t=2.5$ [see Fig. 14(b)], with the system being able to reject it with a slight transient deviation from the reference. Fig. 14(c) shows the response of $i_{\alpha s}$ and $i_{\beta s}$ with their references (allowing to assess the tracking performance of the current controller). As shown, controller (20) adequately operates in low-speed conditions. Moreover, the proposed scheme works well with typical high-performance position servocontrollers.

\section{Comparison With Typical Stationary Frame PI Controller}

A typical solution for stator current control is the use of stationary PI controllers. However, it is widely accepted that they do not deliver high-performance responses, hindering outer control loops. In addition, they are known to yield unacceptable error levels. High-gain stationary PI controllers achieve better results [11], but the inverter commutation frequency poses a fundamental limitation. This factor is crucial when implementing high-performance controllers. In this context, it is remarkable that the stationary frame controller proposed in this paper is capable of delivering a high level of performance.

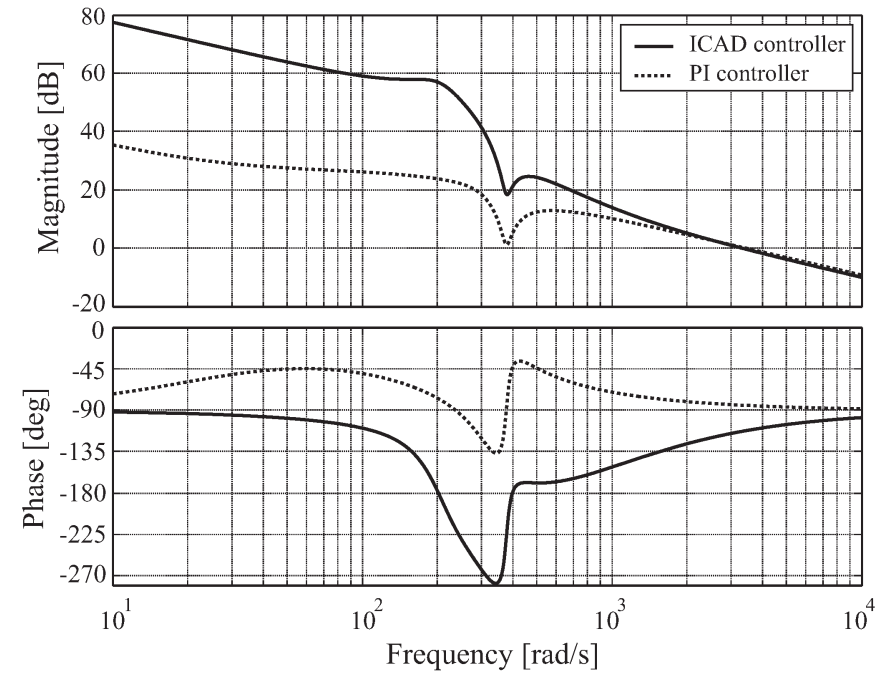

Fig. 15. Comparison between ICAD and PI controllers: Bode diagram of the open-loop individual channels with $\omega_{r}=375 \mathrm{rad} / \mathrm{s}$.

To further this, ICAD controller (20) was compared with a typical PI controller to assess the differences between them.

The most important parameter for the stationary frame current control is the open-loop crossover frequency since it determines the required inverter modulation frequency. As explained in Section V-A, the cross-coupling and rotor element effects can be neglected if a sufficiently high-bandwidth controller is used. To carry out a fair comparison, the PI controller was designed so that its open-loop crossover frequency coincides with that of controller (20). Additionally, both controllers must comply with robustness specifications. This was measured through the phase margin, with a minimum of $45^{\circ}$ being considered. The combination of these constraints yielded the resulting gains $K_{P}=360$ and $K_{I}=9000$.

Fig. 15 shows the Bode plot of the individual channels with each controller $\left(\omega_{r}=375 \mathrm{rad} / \mathrm{s}\right)$. Although both have the same crossover frequency, the PI controller has a much lower openloop gain (dropping near $0 \mathrm{~dB}$ around $375 \mathrm{rad} / \mathrm{s}$ ) due to the resonance peak introduced by the rotor elements and the shaft speed. This indicates that a phase over $-135^{\circ}$ is necessary to ensure robustness. It is not possible to alter $K_{P}$ and $K_{I}$ and keep the same crossover frequency since the phase lead would be lost around $375 \mathrm{rad} / \mathrm{s}$. Conversely, controller (20) yields a high open-loop gain and acceptable stability margins (see Table I).

To highlight the benefits of ensuring an appropriate electrical subsystem control, a simple proportional IFOC speed controller was implemented as follows:

$$
\tau_{\text {ref }}= \begin{cases}\tau_{\max }, & k_{p}\left(\omega_{r, \text { ref }}-\omega_{r}\right)>\tau_{\max } \\ k_{p}\left(\omega_{r, \text { ref }}-\omega_{r}\right), & \left|k_{p}\left(\omega_{r, \text { ref }}-\omega_{r}\right)\right|<\tau_{\max } \\ -\tau_{\max }, & k_{p}\left(\omega_{r, \text { ref }}-\omega_{r}\right)<-\tau_{\max }\end{cases}
$$

where $\tau_{\text {ref }}$ is the torque reference, $\tau_{\max }$ is the maximum torque allowed, $\omega_{r, \text { ref }}$ is the rotor speed reference, and $k_{p}$ is the controller gain. Controller (21) is a saturated control. The saturation element (shown in Fig. 12) prevents high-transient stator currents and inverter overmodulation when operating IFOC 
(a)

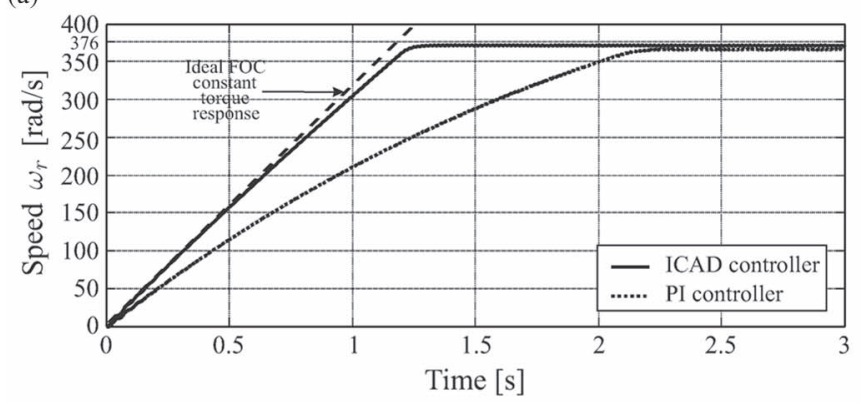

(b)

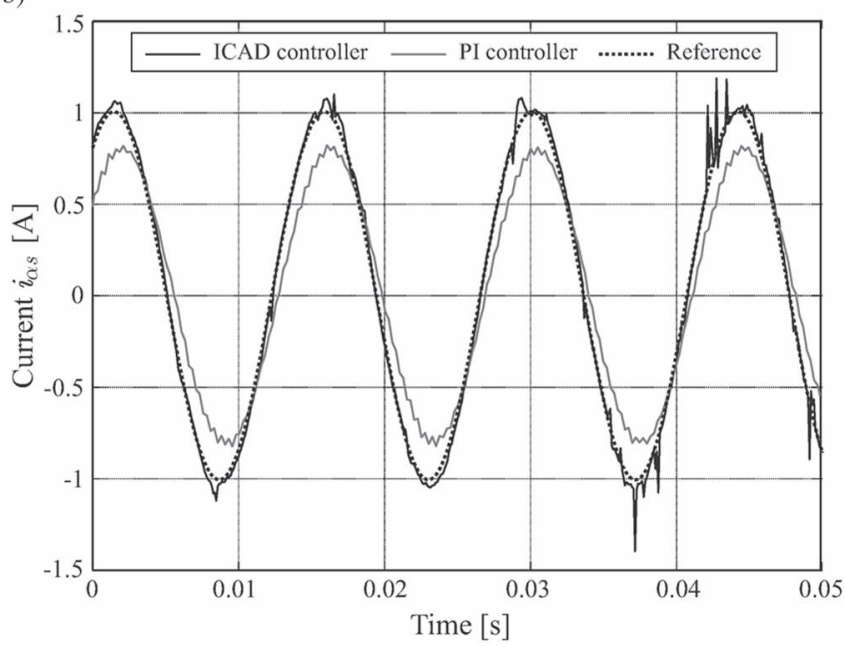

Fig. 16. Comparison between ICAD and PI controllers. Experimental responses: (a) speed $\omega_{r}$ and (b) current $i_{\alpha s}$.

flux-torque controllers with a speed control loop. In this setup, a sudden change in the speed reference (e.g., a large-step startup reference) induces the saturation of the torque reference. As a result, the speed loop opens, and the machine operates with a pure IFOC torque controller and a constant reference $\tau_{\max }$. If low friction is considered and given a properly tuned FOC controller, the rotor speed response during saturation should be a ramp with a constant slope until $\omega_{r}$ is close to $\omega_{r, \text { ref. }}$. Since (21) has no integral effect, a steady-state error will be present.

Fig. 16 compares the experimental responses of the PI and ICAD controllers. The same speed controller and reference (376 rad/s) were used. Fig. 16(a) shows the constant torque slope for an ideally tuned FOC controller assuming a perfect stator current control. As it can be seen, the use of ICAD controller (20) with FOC controller (21) enables a response that is significantly closer to the ideal than when the PI is used, thus rendering superior performance. This highlights the importance of having a correctly designed inner stator current controller as it dramatically affects the performance of the speed controller. If care is not exercised, the torque responses may be affected not only through the effect of shaft friction but also due to the shortcomings of the inner current control loops. Excellent performance would not be achievable without a highperformance stator current control subsystem.

Fig. 16(b) presents the response of current $i_{\alpha s}$ when the PI controller and controller (20) were used. The responses have been superimposed for comparison. It is clear that the PI controller results in greater error levels than those of controller (20), which in turn affect the performance of the FOC speed controller. This was expected from the theoretical predictions in Fig. 15 (from the low open-loop gain of the PI controller) and falls in line with the results reported in the literature for stationary PI control. The comparison carried out in this section shows that, for the same bandwidth, the proposed scheme is able to yield better overall performance without sacrificing robustness.

Figs. 11, 13(c), 14(c), and 16(b) confirm that the proposed controller (20) achieves good tracking and decoupling of the stator currents. This simplifies the outer FOC control loop design by assuming fully controlled stator currents. In turn, the system in (1) and (2) is simplified to a third-order system [25].

Excellent performance was believed to be possible only when synchronous reference frame controllers, decoupling networks, or high-bandwidth stationary controllers (with higher inverter requirements) are employed. Nonetheless, although the proposed controller is stationary and decentralized, it yields high performance. This has been shown through thorough theoretical assessments and confirmed experimentally.

\section{On the Performance of PI CONTROllers}

It could be argued that, by increasing its open-loop gain, a PI controller would improve its performance. As shown in Fig. 15, the system will remain stable for an increase in proportional gain $K_{P}$ since the phase never drops below $-135^{\circ}$; however, there are two possible outcomes. If $K_{P}$ is a common factor in the PI transfer function, the bandwidth would be increased, or if $K_{P}$ is not a common factor, the zero of the PI would tend to zero, reducing the integral action. The reason why the PI exhibits inferior performance when compared with the proposed controller (20) arises from its inability to compensate for the sudden loss of gain around $370 \mathrm{rad} / \mathrm{s}$. This results in a slow response with a large steady-state error for speed references close to $\omega_{r}=370 \mathrm{rad} / \mathrm{s}$ (see Fig. 16).

The selection of PI gains has a major impact on the system performance. To have a benchmark for comparison, the bandwidth has been restricted to assess the potential controller capability within the allowed frequency range. Combinations of PI gains have been obtained by initially setting integral gain $K_{I}$ and then adjusting proportional gain $K_{P}$ so that the desired crossover frequency is obtained. This resulted in a set of PI controllers for a range of integral gains. The Bode plot of the open-loop individual channels is shown in Fig. 17, with the response of controller (20) also included for comparison.

Fig. 17 reveals that, for low integral gains, the open-loop gain is low around $300 \mathrm{rad} / \mathrm{s}$, although the phase remains far from the critical $-180^{\circ}$. By increasing the integral gain, the open-loop gain increases, but the phase margin is reduced. This indicates that a low integral gain ensures high robustness but results in low performance. Conversely, a high integral gain will deliver better performance at the cost of decreasing robustness. For instance, the highest gain PI achieves a high open-loop gain $(20 \mathrm{~dB})$ around $300 \mathrm{rad} / \mathrm{s}$, but in doing so, the phase margin substantially falls, causing an increased overshoot in the time response. Fig. 18 compares the step response of the highest 


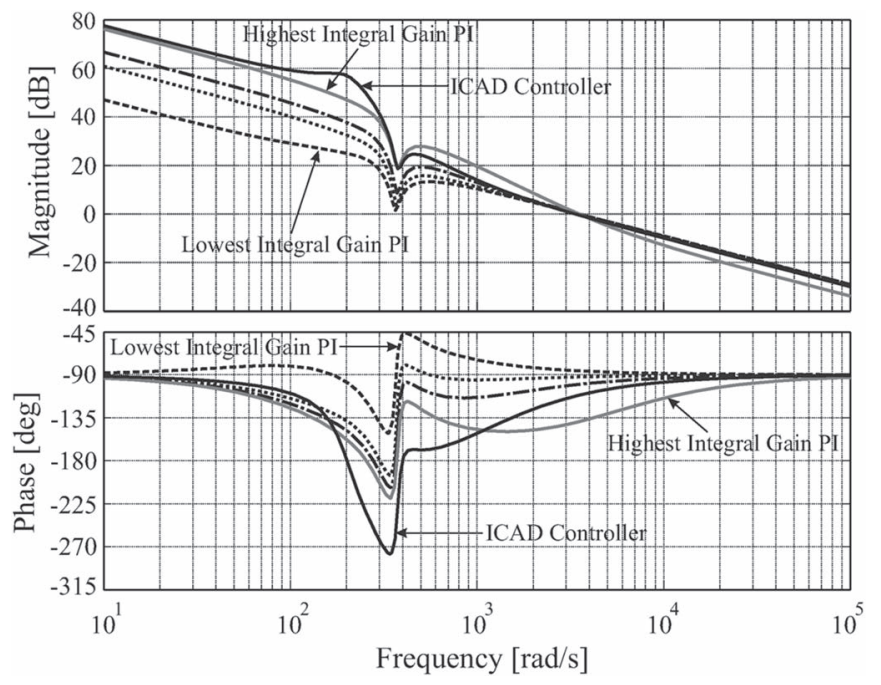

Fig. 17. Comparison between PI controllers with increasing integral gains: Bode diagram of the open-loop channels with $\omega_{r}=375 \mathrm{rad} / \mathrm{s}$.

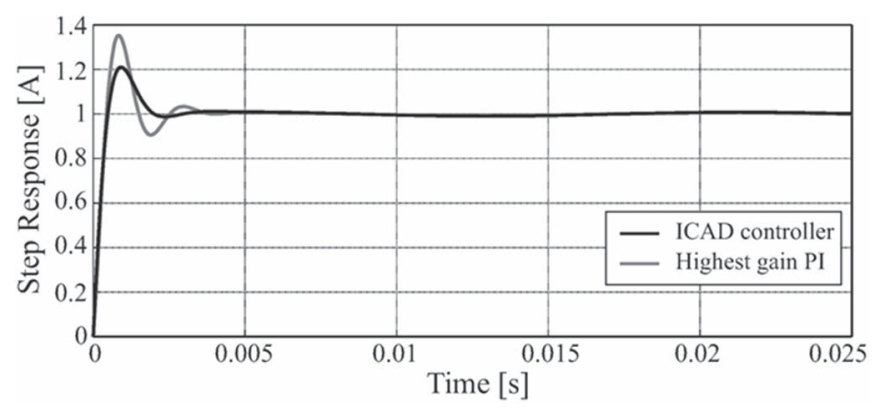

Fig. 18. Step responses with high-integral-gain $\mathrm{PI}$ and ICAD controllers.

gain PI controller with that of controller (20). Although the PI controller eliminates the ringing associated with lower gains around $300 \mathrm{rad} / \mathrm{s}$, the resulting overshoot increases.

Although controller (20) offers superior performance than its stationary PI counterpart, it has a more complex structure. However, the ICAD framework may be employed to evaluate and improve any linear stationary controller. In this line, consider that controller (20) can be expressed as

$$
k_{1}(s)=\frac{326.5(s+1000)}{s} \cdot \frac{(s+400)^{2}}{s^{2}+100 s+42500}
$$

which is effectively a PI controller cascaded with a lag compensator. The contribution of these components in the individual channels can be seen in Fig. 19. Notice that the PI controller enables a similar bandwidth but a greater phase margin than the complete control structure. However, it exhibits a low openloop gain with a phase close to $-180^{\circ}$ around $300 \mathrm{rad} / \mathrm{s}$. These characteristics cause unwanted ringing in the transient response (see Fig. 20). The inclusion of the lag compensator corrects this by increasing the open-loop gain to $\approx 20 \mathrm{~dB}$ at the resonance peak while avoiding a drastic reduction in the phase margin (from $80^{\circ}$ to $67^{\circ}$ ), thus preventing a high overshoot. This effect can be observed in the step response of the individual channels (see Fig. 20). Once the lag compensator is included,

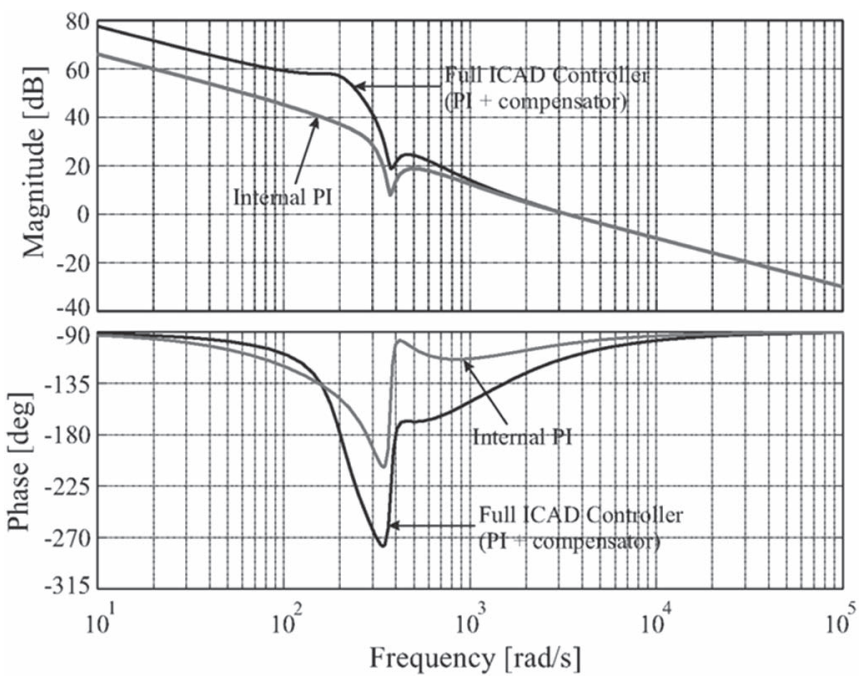

Fig. 19. Bode plots of the open loop individual channels: ICAD controller (20) and its internal PI controller.

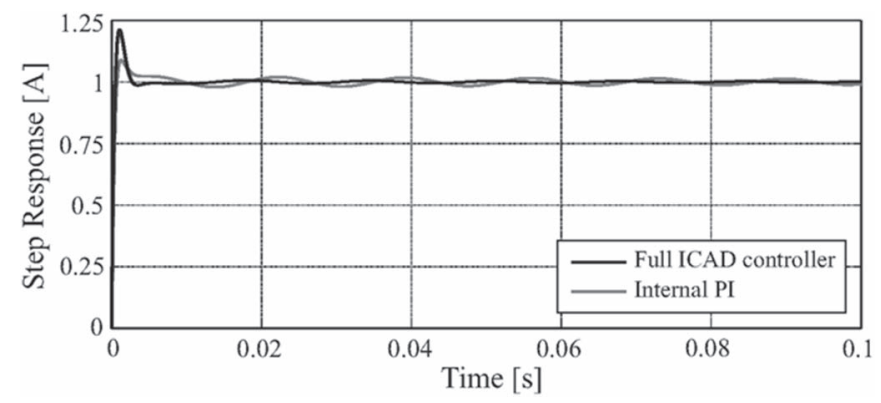

Fig. 20. Simulated step response: ICAD controller (20) and its internal PI controller.

the response exhibits a higher yet tolerable overshoot without the ringing.

It should be stressed that the main contribution of this paper goes beyond obtaining a good controller for an isolated case. The greatest achievement has been the development of a framework for designing and evaluating linear controllers for the stator current subsystem of any IM. Thus, the main advantage of the methodology is not to show a controller that outperforms PI structures but to be able to theoretically analyze the potential and limitations of any linear stationary frame control structure (such as a PI controller) to improve its dynamic behavior. This can be achieved by presenting information to a designer in a simple and direct manner while making use of well-established classical control system tools.

\section{Robustness Considerations}

So far, robustness has been assessed in three ways as follows:

1) The theoretical proof that the system structure is preserved for any valid combination of system parameters and rotor speed, i.e., it is always stable and minimum phase. This is fundamental since it is well known that systems with stable and minimum-phase dynamics are "easy" to control [35]. 


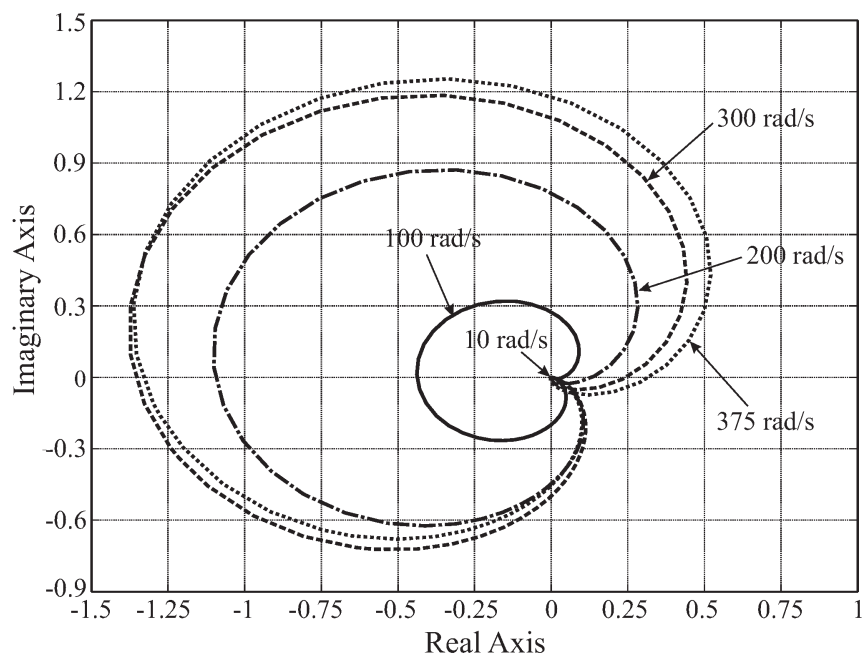

Fig. 21. Nyquist plots of $\gamma(s)$ for the perturbed plant with different $\omega_{r}$.

2) The use of well-known and experimentally proven classical robustness measures based on the Nyquist stability criterion. Normally, classical measures cannot be calculated for MIMO systems; however, ICAD allows doing this. The proposed design complies with typical robustness margins.

3) Experimental validation without "experimental fine tuning," i.e., the control design task was solely based on theoretical analysis and digital simulation tools.

An additional method has been used to complement the aforementioned points. It consists of designing controllers while adopting a perturbed IM model and then experimentally validating the control scheme without said variations. Since the most common variations result from an increase in the resistance due to heat and a reduction in the inductance due to magnetic saturation [36], the following parameters were used (standing for $\approx 100 \%$ of perturbation): $R_{s}=2 R_{s, \text { nom }}$, $R_{r}=2 R_{r, \mathrm{nom}}, L_{s}=0.6 L_{s, \mathrm{nom}}, L_{r}=0.6 L_{r, \mathrm{nom}}$, and $L_{m}=$ $0.59 L_{m, \text { nom. }}$

Let the aforementioned parametric variations be incorporated to the IM model in (1) and (2). Fig. 21 shows the Nyquist plot of $\gamma(s)$ for different $\omega_{r}$. As it can be seen, $\gamma(s)$ does not encircle $(1,0)$. This confirms that the MIMO analysis in Section IV holds for a highly perturbed IM, complementing the results in Fig. 9. As the system complies with conditions (a.1)-(a.3), the design task remains the same as in Section V-C, i.e., the stabilization of the individual channels with a bandwidth higher than the nominal rotor shaft speed; that is, controllers $k_{1}(s)=k_{2}(s)$ being designed as a SISO controller for (7) with $h_{j}(s)=1$ and $i=1$, or explicitly, $g_{11}(s)(1-\gamma(s))$. Controller (20) is able to satisfy the previous conditions.

Fig. 22 shows the Bode plot of the individual channels using controller (20) and the perturbed IM (for $\omega_{r}=375 \mathrm{rad} / \mathrm{s}$ ). The design specifications are clearly met, i.e., a bandwidth of $3700 \mathrm{rad} / \mathrm{s}$, phase and gain margins of $77^{\circ}$ and $20 \mathrm{~dB}$, respectively, and a high open-loop gain around $375 \mathrm{rad} / \mathrm{s}$. The robustness of subsystems $h_{j}(s)$ is evaluated through the frequency response of $k_{j}(s) g_{j j}(s)$ (see Fig. 22). As observed, $h_{j}(s)$ are
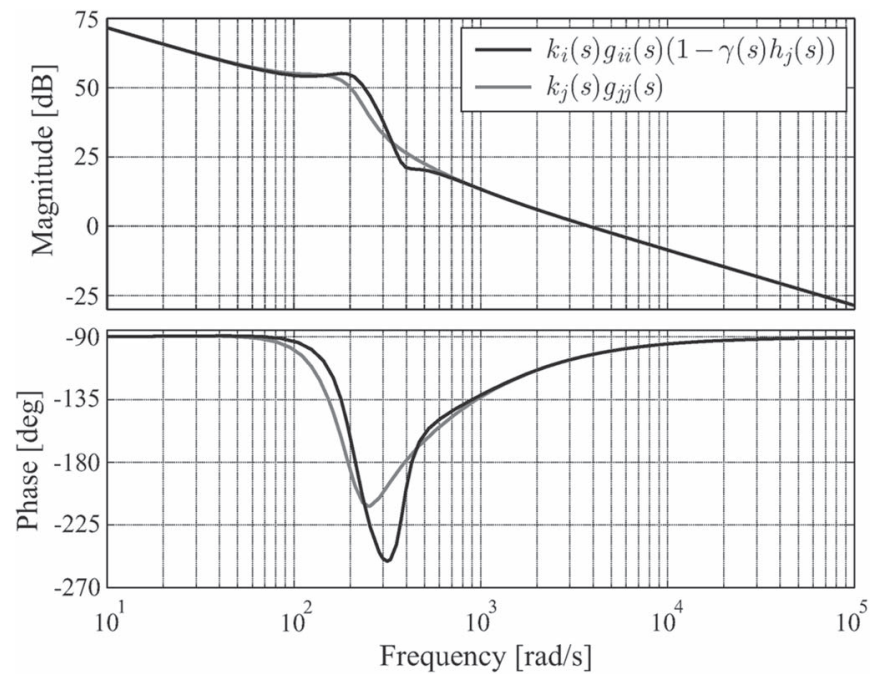

Fig. 22. Bode diagrams of the perturbed open-loop individual channels and elements $k_{j}(s) g_{j j}(s)$ with $\omega_{r}=375 \mathrm{rad} / \mathrm{s}$.

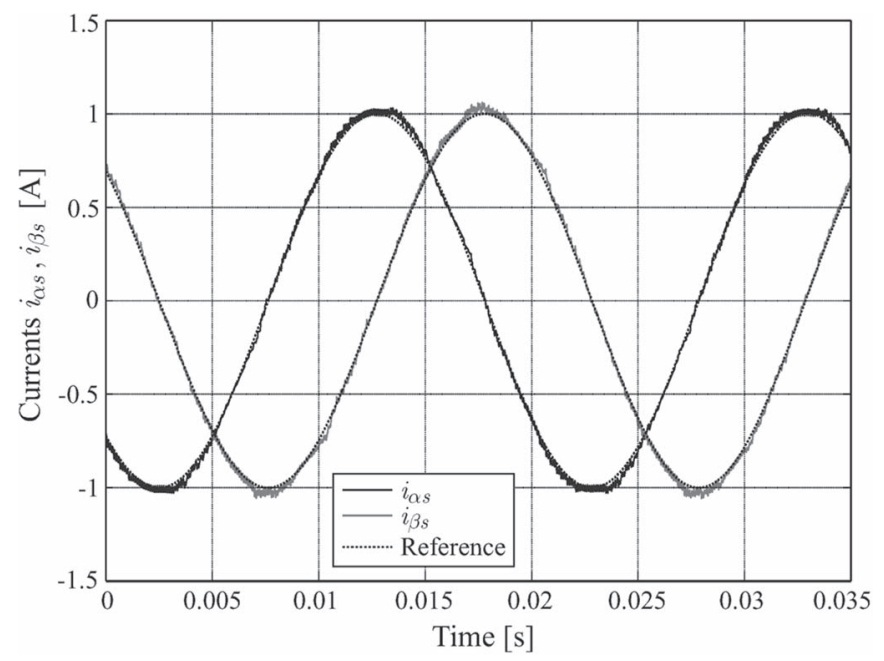

Fig. 23. Simulated response for the perturbed plant: stator currents $i_{\alpha s}$ and $i_{\beta s}$ for a reference signal with a frequency of $300 \mathrm{rad} / \mathrm{s}$.

robust, with phase and gain margins of $77^{\circ}$ and $27 \mathrm{~dB}$, respectively. In addition, the system dynamic structure is preserved, i.e., the Nyquist plot of $\gamma(s) h_{j}(s)$ does not encircle $(1,0)$, with the trajectory being sufficiently far from the critical point (not shown). This analysis shows that robustness requirements (b.1) and (b.2) have been satisfied.

A simulation has been carried out using controller (20) with the perturbed plant. This includes the full nonlinear IM model, the nonlinear characteristics of the mechanical friction, and a two-level VSI with space vector modulation. Fig. 23 shows the stator current response for a reference signal with a frequency of $300 \mathrm{rad} / \mathrm{s}$. It can be noticed that the stator currents closely follow their references with little distortion.

As it has been shown through the theoretical and timedomain assessments in this section, the proposed controller is a good design for a highly perturbed IM, satisfying the performance and robustness specifications. Moreover, the controller also enables high performance when tested with the real unperturbed plant (as presented in Section VI). 


\section{Note On InVERter SWitching Frequency}

It is known that the inverter switching frequency poses bandwidth limitations. Care should be exercised when low switching frequencies are adopted, as done for high-power medium-voltage industrial IM drives. A reduction in the switching frequency may restrict switching losses, albeit at the expense of causing signal delays. These in turn may amplify the cross coupling of the stator current subsystem [12], [13]. The performance of synchronous PI controllers was studied in [16] upon computation and modulation delays. Reference [37] recommends discrete models to capture the effect of pulsewidthmodulation delays.

Although the framework afforded by ICAD has proven successful to design diagonal controllers for plants featuring high cross-coupling dynamics [31]-[33], the effect of time delays associated to low switching frequencies has not been analyzed yet. This opens the door for future investigation.

\section{CONCLUSION}

The control of the stator current subsystem of three-phase IMs for high-performance applications has been thoroughly studied and assessed. Through the framework afforded by ICAD, it has been possible to:

- formally state the requirements for the stability and robustness of stator current decentralized linear control systems in a stationary reference frame;

- design simple decentralized controllers exhibiting a high degree of robustness and performance; and

- measure the cross coupling of the system through the interpretation of ICAD's MSF.

Through the analyses presented in this paper, it has been clearly shown that the stator current subsystem can be controlled using simple, linear, and decentralized controllers in a stationary reference frame and is capable of delivering high performance. For this to be possible, careful attention has to be paid to specific constraints introduced by the multivariable nature of the system, the rotor elements, and the rotor angular speed. By carrying out a truly multivariable analysis, it has been possible to design decoupling decentralized controllers that prevent the use of additional decoupling elements. This simplification of the control system makes the proposed philosophy a suitable option for industrial applications.

The theoretical analysis presented in this paper has been supported by real-time experimental work. Through the experimental results, the importance of achieving an appropriate electrical subsystem control has been elucidated. It has been shown that this directly affects the performance of the outerloop speed or position FOC controllers. The key characteristics of the proposed strategy can be summarized as follows:

- It is a simple design methodology based on classical control.

- It is applicable to any IM.

- It is based on stationary frame coordinates and does not require additional decoupling or signal conditioning elements.
- It is comparable in complexity with stationary frame PI control, but it yields higher performance.

- It is suitable for low- or high-bandwidth requirements.

\section{APPENDIX}

The IM parameters have been obtained following the identification exercise in [34], i.e., $R_{s}=16.2 \Omega, R_{r}=23.2 \Omega$, $L_{s}=1.44 \mathrm{H}, L_{r}=1.5 \mathrm{H}, L_{m}=1.42 \mathrm{H}$, and $P=2$.

\section{References}

[1] J. Chiasson, Modeling and High-Performance Control of Electric Machines. Chichester, U.K.: Wiley, 2005.

[2] J. Rodríguez et al., "High-performance control strategies for electrical drives: An experimental assessment," IEEE Trans. Ind. Electron., vol. 59, no. 2, pp. 812-820, Feb. 2012.

[3] M. Hinkkanen, L. Harnefors, and J. Luomi, "Reduced-order flux observers with stator-resistance adaptation for speed-sensorless induction motor drives," IEEE Trans. Power Electron., vol. 25, no. 5, pp. 1173-1183, May 2010.

[4] J. Lee et al., "Sensorless control of surface-mount permanent-magnet synchronous motors based on a nonlinear observer," IEEE Trans. Power Electron., vol. 25, no. 2, pp. 290-297, Feb. 2010.

[5] C. Huang, C. Wei, J. Yu, and Y. Hu, "Torque and current control of induction motor drives for inverter switching frequency reduction," IEEE Trans. Ind. Electron., vol. 52, no. 5, pp. 1364-1371, Oct. 2005.

[6] M. Mohseni and S. M. Islam, "A new vector-based hysteresis current control scheme for three-phase PWM voltage-source inverters," IEEE Trans. Power Electron., vol. 25, no. 9, pp. 2299-2309, Sep. 2010.

[7] R. Vargas, U. Ammann, B. Hudoffsky, J. Rodríguez, and P. Wheeler, "Predictive torque control of an induction machine fed by a matrix converter with reactive input power control," IEEE Trans. Power Electron., vol. 25, no. 6, pp. 1426-1438, Jun. 2010.

[8] B. Burton, R. G. Harley, G. Diana, and J. L. Rodgerson, "Implementation of a neural network to adaptively identify and control VSI-Fed induction motor stator currents," IEEE Trans. Ind. Appl., vol. 34, no. 3, pp. 580-588, May/Jun. 1998.

[9] D.-C. Lee and Y.-S. Kim, "Control of single-phase-to-three-phase AC/DC/AC PWM converters for induction motor drives," IEEE Trans. Ind. Electron., vol. 54, no. 2, pp. 797-804, Apr. 2007.

[10] R. Krishnan, Electric Motor Drives. Englewood Cliffs, NJ, USA: Prentice-Hall, 2001.

[11] D. G. Holmes, B. P. McGrath, and S. G. Parker, "Current regulation strategies for vector-controlled induction motor drives," IEEE Trans. Ind. Electron., vol. 59, no. 10, pp. 3680-3689, Oct. 2012.

[12] J. Holtz and N. Oikonomou, "Fast dynamic control of medium voltage drives operating at very low switching frequency-An Overview," IEEE Trans. Ind. Electron., vol. 55, no. 3, pp. 1005-1013, Mar. 2008.

[13] J. Holtz et al., "Design of fast and robust current regulators for high-power drives based on complex state variables," IEEE Trans. Ind. Appl., vol. 40, no. 5, pp. 1388-1397, Sep./Oct. 2004.

[14] R. D. Lorenz and D. B. Lawson, "Performance of feedforward current regulators for field-oriented induction machine controllers," IEEE Trans. Ind. Appl., vol. IA-23, no. 4, pp. 597-602, Jul. 1987.

[15] F. Briz del Blanco, M. W. Degner, and R. D. Lorenz, "Dynamic analysis of current regulators for AC motors using complex vectors," IEEE Trans. Ind. Appl., vol. 35, no. 6, pp. 1424-1432, Nov./Dec. 1999.

[16] A. G. Yepes, A. Vidal, J. Malvar, O. López, and J. Doval, "Tuning method aimed at optimized settling time and overshoot for synchronous proportional-integral current control in electric machines," IEEE Trans. Power Electron., vol. 29, no. 6, pp. 3041-3054, Jun. 2014.

[17] F. D. Freijedo et al., "Tuning of synchronous-frame PI current controllers in grid-connected converters operating at a low sampling rate by MIMO root locus," IEEE Trans. Ind. Electron., vol. 62, no. 8, pp. 5006-5017, Aug. 2015.

[18] M. Comanescu, L. Xu, and T. D. Batzel, "Decoupled current control of sensorless induction-motor drives by integral sliding mode," IEEE Trans. Ind. Electron., vol. 55, no. 11, pp. 3836-3845, Nov. 2008.

[19] L. Harnefors and H.-P. Nee, "Model-based current control of AC machines using the internal model control method," IEEE Trans. Ind. Appl., vol. 34, no. 1, pp. 133-141, Jan./Feb. 1998. 
[20] B. Bahrani, S. Kenzelmann, and A. Rufer, "Multivariable-PI-based dq current control of voltage source converters with superior axis decoupling capability," IEEE Trans. Ind. Electron., vol. 58, no. 7, pp. 3016-3026, Jul. 2011.

[21] S. Li, T. A. Haskew, R. P. Swatloski, and W. Gathings, "Optimal and direct-current vector control of direct-driven PMSG wind turbines," IEEE Trans. Power Electron., vol. 27, no. 5, pp. 2325-2337, May 2012.

[22] L. Amézquita-Brooks, E. Licéaga-Castro, and J. Licéaga-Castro, "The structural robustness of the induction motor stator currents subsystem," Asian J. Control, vol. 16, no. 6, pp. 1632-1645, Nov. 2014.

[23] C. E. Ugalde-Loo, L. A. Amézquita-Brooks, E. Licéaga-Castro, and J. Licéaga-Castro, "Structural robustness assessment of electric machine applications using individual channel analysis and design," Cybern. Phys., vol. 2, no. 2, pp. 108-118, Sep. 2013.

[24] L. Amézquita-Brooks, E. Licéaga-Castro, and J. Licéaga-Castro, "Novel design model for the stator currents subsystem of induction motors," Appl. Math. Model., vol. 38, no. 23, pp. 5623-5634, Dec. 2014.

[25] L. Amézquita-Brooks, J. Licéaga-Castro, and E. Licéaga-Castro, "Speed and position controllers using indirect field-oriented control: A classical control approach," IEEE Trans. Ind. Electron., vol. 61, no. 4, pp. 1928-1943, Apr. 2014.

[26] J. O'Reilly and W. E. Leithead, "Multivariable control by 'individual channel design'," Int. J. Control, vol. 54, no. 1, pp. 1-46, 1991.

[27] J. Licéaga, E. Licéaga, and L. Amézquita, "Multivariable gyroscope control by individual channel design," in Proc. IEEE Int. CCA, Toronto, ON, Canada, Aug. 2005, pp. 785-790.

[28] C. E. Ugalde-Loo, E. Acha, and E. Licéaga-Castro, "Fundamental analysis of the electromechanical oscillation damping control loop of the static VAr compensator using individual channel analysis and design," IEEE Trans. Power Del., vol. 25, no. 4, pp. 3053-3069, Oct. 2010.

[29] C. E. Ugalde-Loo, L. Amézquita-Brooks, E. Licéaga-Castro, and J. Licéaga-Castro, "Analysis and efficient control design for generatorside converters of PMSG-based wind and tidal stream turbines," in Proc. 18th PSCC, Wroclaw, Poland, Aug. 2014, pp. 1-7.

[30] H. K. Khalil, Nonlinear Systems. Upper Saddle River, NJ, USA: Pearson, 2002

[31] E. Liceaga-Castro, J. Liceaga-Castro, and C. E. Ugalde-Loo, "Beyond the existence of diagonal controllers: From the relative gain array to the multivariable structure function," in Proc. 44th IEEE CDC-ECC, Seville, Spain, Dec. 2005, pp. 7150-7156.

[32] C. E. Ugalde-Loo, "Dynamical modelling of power systems with power electronic controllers using individual channel analysis and design." $\mathrm{Ph} . \mathrm{D}$. dissertation, College Sci. Eng., Univ. Glasgow, Glasgow, U.K., 2009.

[33] L. A. Amézquita-Brooks, C. E. Ugalde-Loo, E. Licéaga-Castro, and J. Licéaga-Castro, "The multivariable structure function as an extension of the RGA matrix: Relationship and advantages," Cybern. Phys., vol. 2, no. 2, pp. 53-62, Sep. 2013.

[34] L. Amézquita-Brooks, J. Licéaga-Castro, and E. Licéaga-Castro, "Induction motor identification for high performance control design," Int. Rev. Elect. Eng., vol. 4, no. 5, pp. 825-836, Sep./Oct. 2009.

[35] J. C. Doyle, B. A. Francis, and A. R. Tannenbaum, Feedback Control Theory. New York, NY, USA: Macmillan, 1992.

[36] R. T. Novotnak, J. Chiasson, and M. Bodson, "High-performance motion control of an induction motor with magnetic saturation," IEEE Trans. Control Syst. Technol., vol. 7, no. 3, pp. 315-327, May 1999.

[37] H. Kim, M. W. Degner, J. M. Guerrero, F. Briz, and R. D. Lorenz, "Discrete-time current regulator design for AC machine drives," IEEE Trans. Ind. Appl., vol. 46, no. 4, pp. 1425-1435, Jul./Aug. 2010.

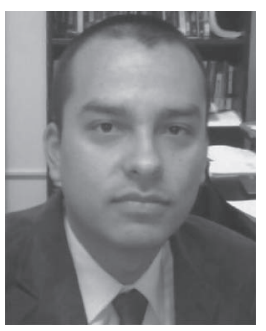

Luis A. Amézquita-Brooks (M'13) was born in México City, México. He received the B.Eng. degree in electronic systems, the M.Sc. degree in control and automation, and the Ph.D. degree in autonomous systems from the Monterrey Institute of Technology and Higher Education (ITESM), Campus Estado de México, México, in 2003, 2005, and 2010, respectively.

$\mathrm{He}$ is currently a Full-Time Professor with the Facultad de Ingeniería Mecánica y Eléctrica, Universidad Autónoma de Nuevo León,

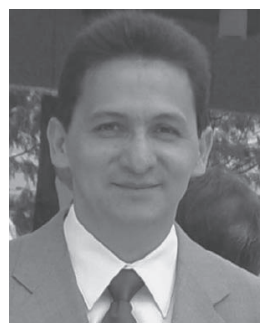

Jesús Licéaga-Castro was born in México City, México. He received the B.Eng. degree in electronic engineering from the Iberoamericana University, México City, México, in 1985, the M.Sc. degree in automatic control from the $\mathrm{Na}$ tional Polytechnic Institute (IPN), México City, México, in 1988, and the Ph.D. degree in electronics and electrical engineering from the University of Glasgow, Glasgow, U.K., in 1995.

He was a Full-Time Professor with and the Leader of the Electric Machines Control Research Group, Monterrey Institute of Technology and Higher Education (ITESM), Campus Estado de México, México. He is currently a Full-Time Professor with the Department of Electronic Engineering, Universidad Autónoma Metropolitana, Azcapotzalco, Reynosa Tamaulipas, Ciudad de México, D.F., México.

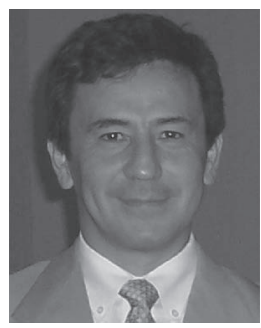

Eduardo Licéaga-Castro (M'04) was born in México City, México. He received the B.Eng. degree in aerospace engineering and the M.Sc. degree in automatic control from the National Polytechnic Institute (IPN), México City, México, in 1983 and 1984, respectively, and the Ph.D. degree in aerospace engineering from the University of Glasgow, Glasgow, U.K., in 1988.

$\mathrm{He}$ has held academic posts with the Universidad Carlos III de Madrid, Leganes, Spain; IPN; Glasgow Caledonian University, Glasgow, U.K.; the University of Glasgow; and the University of Strathclyde, Glasgow, U.K. He has also held industrial research posts with Daimler Chrysler, Berlin, Germany, and with Arquimea, Madrid, Spain. He is currently a Full-Time Professor with the Facultad de Ingeniería Mecánica y Eléctrica, Universidad Autónoma de Nuevo León, San Nicolás de los Garza, Nuevo León, México.

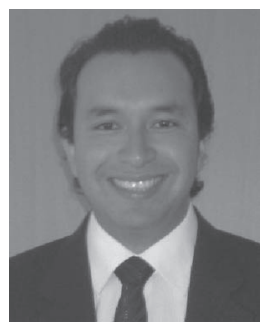

Carlos E. Ugalde-Loo (M'02) was born in México City, México. He received the B.Sc. degree in electronics and communications engineering from the Monterrey Institute of Technology and Higher Education (ITESM), Ciudad de México, México, in 2002, the M.Sc. degree in electrical engineering from the National Polytechnic Institute (IPN), México City, México, in 2005, and the Ph.D. degree in electronics and electrical engineering from the University of Glasgow, Glasgow, U.K., in 2009.

In 2010, he joined the Cardiff School of Engineering, Cardiff University, Cardiff, U.K., where he is currently a Lecturer in electrical power systems. 